\title{
Quasi-accord de phase et interactions non linéaires en optique intégrée
}

\author{
M. De Micheli \\ Laboratoire de Physique de la Matière Condensée, UMR 6622, Université \\ de Nice-Sophia Antipolis, Parc Valrose, 06108 Nice cedex 2, France
}

\begin{abstract}
Résumé : Ces dernières années, l'optique non linéaire a été bouleversée par la mise au point de procédés permettant d'obtenir des matériaux présentant une modulation périodique du coefficient non linéaire. Dans ce chapitre, nous décrirons ces techniques et leurs combinaisons avec les techniques de l'optique intégrée, ce qui permet de concevoir un grand nombre de nouveaux composants, comme des sources laser accordables miniatures.
\end{abstract}

\section{INTRODUCTION}

Ces dernières années, un effort de recherche considérable a porté sur la réalisation de sources cohérentes solides accordables. La mise au point de techniques permettant d'obtenir des cristaux ferroélectriques polarisés périodiquement [1] a permis d'utiliser de manière très efficace la technique de quasi-accord de phase proposée par N. Blombergen en 1962 [2]. Cette technique présente de nombreux avantages. Tout d'abord, elle permet d'étendre la plage spectrale d'utilisation de matériaux non linéaires bien connus comme $\mathrm{LiNbO}_{3}$ et $\mathrm{KTP}$, en donnant en plus accès à leurs coefficients non linéaires les plus élevés. Deuxièmement, elle permet d'utiliser des matériaux non linéaires non biréfringents comme l'AsGa. Enfin, elle permet d'introduire en différents endroits d'un même substrat non linéaires des propriétés d'accord de phase différentes, ce qui permet de concevoir des composants entièrement nouveaux. Plusieurs processus non linéaires $[3,4]$ ont été démontrés dans cette configuration et en particulier l'oscillation paramétrique $[5,6]$.

La réalisation de guides d'ondes dans les cristaux polarisés périodiquement est un enjeu important car cette configuration permet de profiter d'un confinement de l'énergie plus important, de longueurs d'interaction plus grandes et de la possibilité d'ajuster l'accord en utilisant l'effet électro-optique.

Dans ce cours, nous commencerons donc par étudier le quasi-accord de phase et nous présenterons quelques réalisations dans des cristaux massifs qui permettent d'illustrer l'état de l'art en 1998 ainsi que les possibilités originales de la configuration de quasi-accord de phase. Dans une deuxième partie, nous nous concentrerons sur les interactions en optique guidée en nous intéressant plus particulièrement aux guides d'ondes réalisés par échange protonique (PE) $[7,8]$ sur niobate de lithium polarisé périodiquement (PPLN) dans lesquels ont été réalisées la majorité des expériences. Cette partie comprendra donc une présentation des 
difficultés technologiques rencontrées pour réaliser des guides qui respectent à la fois la non-linéarité du cristal et l'organisation périodique des domaines.

\section{QUASI-ACCORD DE PHASE}

\subsection{Principe}

Cette technique d'accord de phase a été initialement proposée par $\mathbf{N}$. Bloembergen [2] en 1962. Elle s'applique à tous les processus d'interaction paramétrique en $\chi^{2}$, mais elle est plus simple à exposer dans le cas de la génération de second harmonique. C'est donc dans ce cas que nous nous placerons, en rappelant tout d'abord qu'après une longueur d'interaction $L$, la puissance harmonique est donnée par :

$$
P_{2 \omega}(L)=P_{\omega}^{2}\left|\int_{0}^{L} I_{R} e^{i \varepsilon(\omega) \cdot z} d d\right|^{2}
$$

Dans cette expression, $P_{\omega}$ est la puissance fondamentale incidente, $I_{R}$ est l'intégrale de recouvrement entre les champs des ondes en interaction et le coefficient non linéaire $d(x, y) d u$ matériau, et $\delta k(\omega)=k(2 \omega)-2 k(\omega)$ est le désaccord de phase. Après intégration le long de la direction de propagation, le terme $\mathrm{e}^{\mathrm{i} \delta \mathrm{k}(\omega) \cdot \mathrm{x}}$ donne le facteur en $\operatorname{sinc}^{2}\left(\frac{\delta k(\omega) . L}{2}\right)$ qui indique que les seules interactions efficaces sont obtenues autour de la fréquence fondamentale $\omega$ pour laquelle $\delta k(\omega)=0$. Cette condition, dite condition d'accord de phase, correspond au cas où les deux ondes, fondamentale et harmonique, se propagent à la même vitesse. Cette condition est généralement obtenue en utilisant des matériaux non linéaires biréfringents, les ondes en interaction étant polarisées à $90^{\circ}$ et voyant des indices différents. Le Quasi-Accord de Phase (QAP) consiste à introduire une périodicité associée à un vecteur d'onde $\mathrm{K}$, dans l'un des facteurs de l'intégrale $I_{R}$, de manière à compenser le désaccord de phase. L'efficacité de conversion est alors maximale pour la fréquence $\omega$ telle que $\delta k(\omega)-K=0$ pour laquelle on satisfait une condition de quasi-accord de phase.

Cette périodicité peut être introduite en modulant l'indice du matériau [9] ce qui a pour conséquence de moduler, avec la même période, la forme des champs des ondes en interaction et donc l'intégrale de recouvrement $I_{R}$. Ce procédé a cependant l'inconvénient de créer un réseau qui a la possibilité de coupler les modes guidés avec les modes de rayonnement introduisant ainsi des pertes à la propagation qui limitent sérieusement l'intérêt de la technique.

Cette périodicité peut également être introduite en modulant le coefficient non linéaire $d$. Une situation facile à imaginer est celle qui consiste à inverser périodiquement le signe de ce coefficient. En fonction de la direction de propagation $z$, il prend donc la forme d'une fonction créneau qui peut se développer en série de Fourier suivant :

$$
d(z)=\sum_{n=0}^{+\infty} d_{n} e^{-i n K z} \quad \text { avec } \quad d_{0}=(2 a-1) d_{33} \quad \text { et } \quad d_{n}=\frac{2 d_{33}}{n \pi} \sin (n \pi a)
$$

où $a$ est le facteur de forme compris entre 0 et 1 et défini par le rapport entre la largeur du créneau et la période et $d_{33}$ est un élément du tenseur $\chi^{(2)}$. 
Il y donc autant de situations de quasi-accord de phase qu'il y a de solutions à l'équation

$$
\delta k(\omega)-n K=0 .
$$

D'après l'équation (2), on voit que pour une fréquence $\omega$ fixée, la situation la plus favorable est celle pour laquelle (3) est vérifiée au premier ordre $(n=1)$ avec un facteur de forme

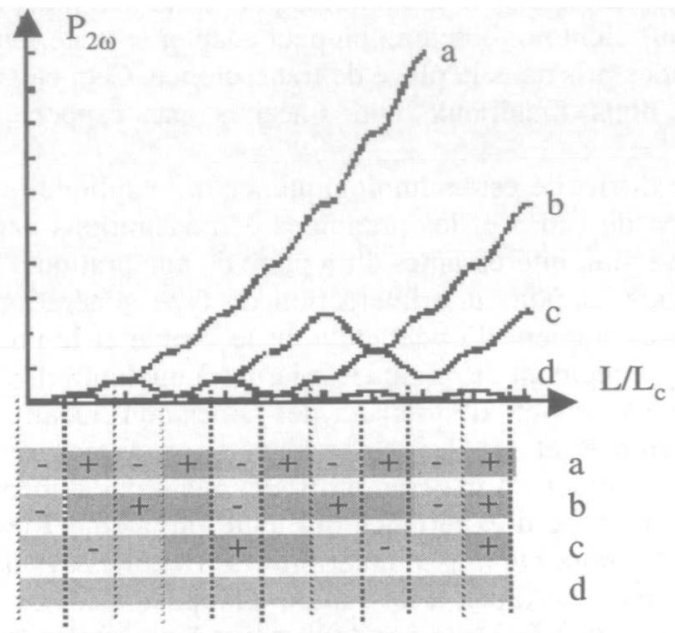

Figure 2.1 : Puissance harmonique (unité arbitraire) générée en fonction de la longueur de l'échantillon (exprimée en multiple de la longueur de cohérence) et de l'ordre de quasi-accord de phase. Les différentes courbes correspondent à :

(a) : [n=1, a=0,5], (b) : [n=2, a=0,25], (c) : [n=3, a=0,5], (d) : [n=0].

$a=0,5$. Mais, pour toutes les valeurs de $n$ et en choisissant le facteur de forme de manière à optimiser $d_{n}$, il est possible d'obtenir un signal de second harmonique proportionnel à $L^{2}$, le coefficient de proportionnalité variant en $\frac{1}{n^{2}}$ (figure 2.1). On voit également sur cette figure, que la configuration la plus favorable, le quasi-accord de phase au premier ordre, correspond à la situation où le signe du coefficient non linéaire change chaque fois que le déphasage accumulé au cours de la propagation est égal à $\pi$. La longueur ainsi définie est la longueur de cohérence.

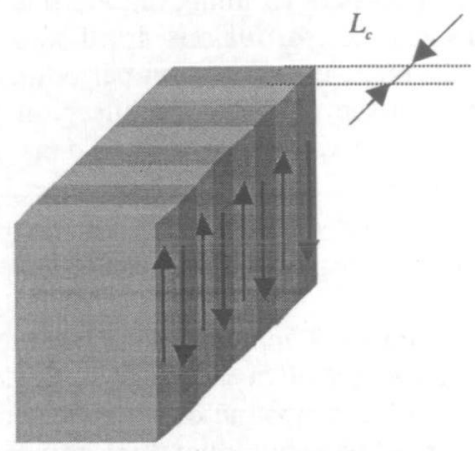

Figure 2.2 : Cristal artificiel présentant une inversion périodique du signe du coefficient non linéaire. 
D'un point de vue pratique, la possibilité d'obtenir un signal de deuxième harmonique important en satisfaisant une condition de quasi-accord de phase est extrêmement intéressante. En effet, elle permet d'utiliser des ondes présentant la même polarisation, ce qui rend utilisables les matériaux non linéaires non biréfringents comme l'AsGa et les coefficients non linéaires diagonaux qui sont souvent les plus forts. De plus, en choisissant la période de modulation du coefficient non linéaire, on peut adapter le matériau à n'importe quel ensemble de longueurs d'ondes pris dans la plage de transparence. Ceci étend considérablement la plage d'utilisation des bons matériaux non linéaires par rapport à l'accord de phase par biréfringence.

La principale difficulté est technologique, ce qui explique le délai de près de 30 années entre la publication de l'idée et les premières démonstrations expérimentales présentant des efficacités de conversion intéressantes d'un point de vue pratique. En effet, dans les matériaux non linéaires connus, et pour une interaction du type génération de deuxième harmonique dans une gamme de longueur d'onde couvrant le visible et le proche infrarouge, la longueur de cohérence est typiquement de quelques microns à quelques dizaines de microns. Il est donc très difficile, voire impossible de préparer des lames non linéaires ayant pour épaisseur cette longueur de cohérence et de les assembler après avoir périodiquement renversé leur orientation, pour constituer un matériau artificiel ayant des dimensions intéressantes pour les applications. C'est ce type d'expérience qui a été initialement tenté pour vérifier la théorie, mais il n'a jamais été possible d'assembler plus de 10 lames ayant des épaisseurs de plusieurs longueurs de cohérence de façon à les rendre manipulables. Les taux de conversion obtenus étaient donc beaucoup trop faibles pour donner lieu à des applications pratiques [10].

\subsection{Techniques d'inversion de domaines}

Il en est tout autrement aujourd'hui, depuis que l'on a montré que dans les matériaux ferroélectriques, il était possible d'introduire sur plusieurs centimètres de long, une inversion périodique du signe du coefficient non linéaire en renversant périodiquement la polarisation spontanée de ces matériaux. Plusieurs solutions ont été proposées et testées. Il s'agit principalement de diffusion ou d'échange ionique et d'application d'un champ électrique périodique. Dans tous ces cas, on utilise un masque défini par photolithographie, ce qui permet de répéter des motifs de quelques microns sur des substrats de plusieurs centimètres carrés. Deux familles de matériaux ont été plus particulièrement utilisées. Il s'agit d'une part, du KTP et des cristaux dérivés, KTA, RTA, ..., d'autre part du $\mathrm{LiNbO}_{3}$ et du $\mathrm{LiTaO}_{3}$.

Historiquement, les premiers résultats ont été obtenus en optique intégrée et en utilisant des échanges ioniques ou des diffusions localisées qui permettent d'obtenir de faibles volumes de cristaux présentant une inversion périodique du signe du coefficient non linéaire. Sur KTP, la technique utilisée consiste à réaliser un guide d'onde segmenté en plongeant pendant $90 \mathrm{mn}$ le cristal recouvert d'un masque dans un mélange de $\mathrm{RbNO}_{3}(99 \mathrm{M} \%)$ et de $\mathrm{Ba}\left(\mathrm{NO}_{3}\right)_{2}(1 \mathrm{M} \%)$ porté à $360^{\circ} \mathrm{C}$. Cela permet d'obtenir une augmentation d'indice due à un échange $\mathrm{K}^{+} \leftrightarrow \mathrm{Rb}^{+}$et la présence de $\mathrm{Ba}$ permet d'inverser le signe de la polarisation et donc le coefficient non linéaire dans les zones échangées [11]. Le composant obtenu est représenté sur la figure 2.3 .

Sur $\mathrm{LiNbO}_{3}$, les premiers échantillons présentant une inversion périodique du coefficient non linéaire ont été réalisés en diffusant à $1000^{\circ} \mathrm{C}$, des rubans de titane sur la face $Z^{*}$ du cristal [12]. Ce processus s'accompagne d'un renversement de la polarisation dans le volume diffusé. Les domaines ainsi formés prennent une forme triangulaire ou trapézoïdale suivant la valeur des différents paramètres et pénètrent jusqu'à quelques microns de profondeur. La concentration nécessaire pour obtenir ce renversement est faible et ne crée pas de modulation 
d'indice décelable. On peut donc ensuite utiliser l'échange protonique [13] qui ne requiert qu'une faible élévation de température $\left(300^{\circ} \mathrm{C}\right)$ et permet de créer un guide d'onde sans effacer les domaines [14].

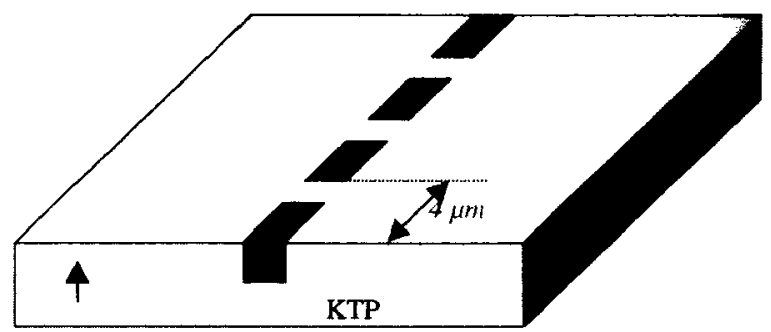

Figure 2.3 : Guide segmenté et polarisé périodiquement sur KTP adapté au doublage en fréquence des diodes laser

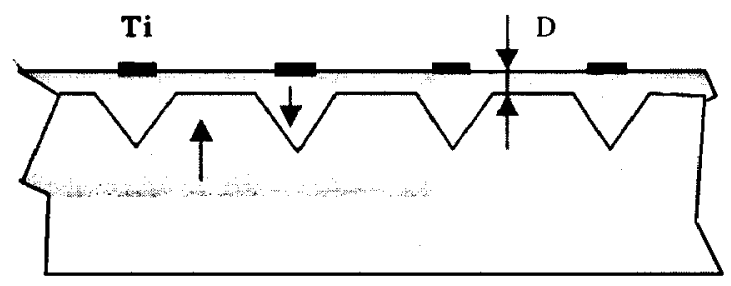

\author{
Guide d'onde \\ réalisé par \\ échange protonique
}

Figure 2.4 : Profil des domaines induits par diffusion de Ti dans le niobate de lithium.

À ces techniques faisant appel à la diffusion ou à l'échange ionique on tend à préférer aujourd'hui celles qui reposent sur l'application d'un champ électrique périodique [15] et qui s'adaptent, en changeant la valeur des paramètres et quelques détails, aux deux familles de matériaux. Le principe du dispositif de polarisation est représenté sur la figure 2.5 . Le champ est appliqué de part et d'autre du substrat en utilisant des électrodes liquides pour éviter au maximum les problèmes liés aux effets de bord puisque les champs à appliquer sont de plusieurs $\mathrm{kV}$. La périodicité est créée en définissant sur une des faces du substrat un masque diélectrique isolant qui gêne l'établissement du courant de polarisation dans les régions qu'il recouvre. Pour obtenir un renversement de la polarisation il faut appliquer au cristal un champ supérieur au champ coercitif $\left(5 \mathrm{kV} / \mathrm{mm}\right.$ pour $\mathrm{KTP}$ et $21 \mathrm{kV} / \mathrm{mm}$ pour $\mathrm{LiNbO}_{3}$ ). Dans les parties en contact avec l'électrode, il se crée alors des domaines en forme d'aiguille présentant une polarisation opposée à la polarisation initiale et qui traversent tout le substrat avant de s'élargir avec des parois quasi perpendiculaires à la surface. En contrôlant précisément la valeur du champ appliqué, la durée d'application du champ et divers autres paramètres comme l'état de surface, il est possible d'obtenir des substrats présentant des domaines alternativement polarisés qui reproduisent fidèlement le masque initial [16]. Cependant, le masque diélectrique ne crée une périodicité du champ que sur quelques $\mu \mathrm{m}$ de profondeur, profondeur qui est d'autant plus faible que la période est courte. Au-delà, le cristal voit un champ parfaitement homogène. De plus, au cours de la propagation, les parois des domaines peuvent être déviées par les défauts présents dans le cristal. Si l'on ajoute à cela les difficultés liées à la manipulation de champs de plusieurs dizaines de $\mathrm{kV}$, on comprend parfaitement que l'épaisseur des substrats présentant une polarisation périodique soit limitée. Aujourd'hui, les 
substrats de PPLN (Periodically Poled Lithium Niobate) disponibles commercialement font $500 \mu \mathrm{m}$ d'épaisseur pour des périodes supérieures à $6 \mu \mathrm{m}$ et en laboratoire, on a atteint $1 \mathrm{~mm}$. Pour le doublage des diodes lasers à $0,8 \mu \mathrm{m}$, des périodes plus courtes sont nécessaires, et les substrats utilisés font en général $300 \mu \mathrm{m}$.

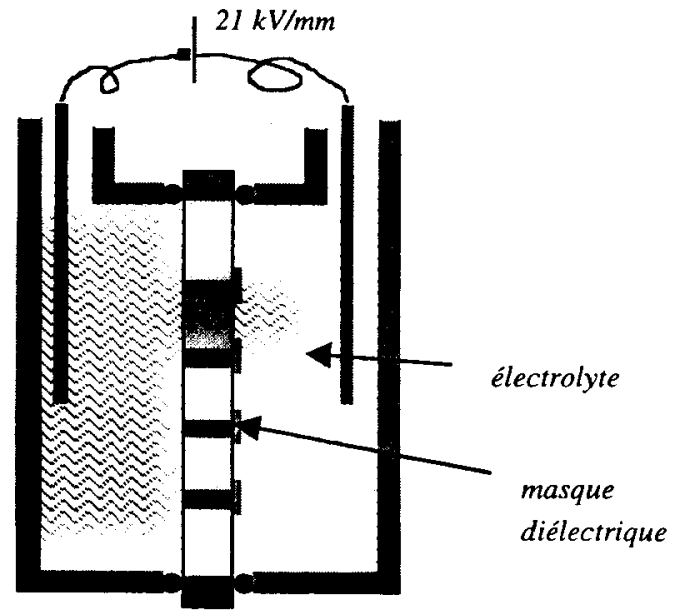

Figure 2.5 : Schéma de principe de la cellule de polarisation par champ électrique

\section{3 État de l'art et réalisations}

Initié au début des années 1990 , avec la publication des premiers résultats expérimentaux sur niobate de lithium polarisé périodiquement présentant des efficacités de conversion importantes, le domaine des interactions non linéaires en configuration de quasi-accord de phase est aujourd'hui en pleine explosion. Il est donc très difficile de faire un état de l'art du domaine, et celui-ci a de fortes chances d'être périmé avant d'être imprimé. Le paragraphe suivant est donc plus une photo du domaine prise en mai 1998, puisque les réalisations choisies ont pour la plupart été présentées à CLEO'98. Dans cette sélection, une place particulière est faite aux réalisations qui soulignent les spécificités de la configuration de quasi-accord de phase. Un domaine que l'apparition des cristaux périodiquement polarisés a considérablement modifié est très certainement celui des oscillateurs paramétriques optiques (OPO). Les efficacités de conversion disponibles ont permis en particulier de réaliser de nombreux OPO continus en plaçant le cristal dans la cavité du laser de pompe [17] ou dans une cavité externe linéaire ou en anneau [18]. Le pompage par diode est également devenu possible en utilisant du PPLN [19, 20] ou du PPLT [21]. Les qualités spectrales des ondes signal et idler peuvent également être grandement améliorées, et il est possible d'utiliser ces OPO pour la spectroscopie [22]. Les matériaux polarisés périodiquement sont également utilisés avec succès pour la conversion de fréquence vers le bleu et l'ultra-violet en configuration massive ou en guides d'ondes. À côté de ces réalisations où la technique de polarisation périodique permet de réaliser des composants plus performants que les autres techniques d'accord de phase, il y a également plusieurs expériences qui mettent en évidence les possibilités nouvelles offertes par la technique de QAP [23]. En effet, il est possible par 
photolithographie de définir plusieurs périodes sur le même cristal, offrant ainsi la possibilité de changer la longueur d'onde d'accord de phase en translatant le cristal. Ces périodes différentes peuvent également être placées à la suite les unes des autres ce qui permet de réaliser plusieurs interactions non linéaires en cascade [24]. Il est également possible de réaliser un substrat sur lequel la période d'inversion évolue continuellement le long de la direction de propagation. Il est ainsi possible de doubler des sources ayant un spectre large comme les impulsions ultra-courtes [25], et même, en adaptant soigneusement la variation de la période à la dispersion du matériau et aux caractéristiques de l'impulsion incidente (spectre et décalage temporel des fréquences) il est possible de comprimer cette impulsion [26].

\section{INTERACTIONS NON LINÉAIRES EN OPTIQUE GUIDÉE}

\subsection{Intérêt et spécificité des guides d'ondes en optique non linéaire}

En optique non linéaire, la configuration guidée présente plusieurs avantages et impose quelques conditions supplémentaires que nous allons passer en revue dans ce paragraphe.

Le premier avantage de l'optique intégrée est certainement celui du confinement de l'énergie [27] qui permet d'obtenir des efficacités importantes avec des puissances de pompe faibles ou, pour une puissance de pompe donnée, permet d'augmenter très sensiblement l'efficacité de conversion. Dans le cas de la génération de second harmonique, cette augmentation peut atteindre deux ordres de grandeurs. Un autre avantage de l'optique intégrée est de satisfaire les conditions d'accord de phase ou de quasi-accord de phase [28] entre les indices effectifs des modes et non pas entre les indices du matériau. En jouant avec le profil d'indice du guide, il est possible d'utiliser différents modes guidés [29] et même des modes de rayonnement [30] pour satisfaire les conditions de conservation. Cependant, il ne faut pas oublier que pour que l'interaction reste efficace, il faut également obtenir un bon recouvrement entre les modes en interaction et le matériau non linéaire. La forme particulière des distributions de champ des différents modes limite en fait sévèrement le nombre des combinaisons efficaces.

La figure 3.1 illustre l'amélioration du confinement obtenu en configuration guidée. Dans le matériau massif, le faisceau est focalisé au centre du cristal, puis diverge. L'optimisation consiste à adapter la section minimale $S$ du faisceau à la longueur $L$ du cristal pour se placer

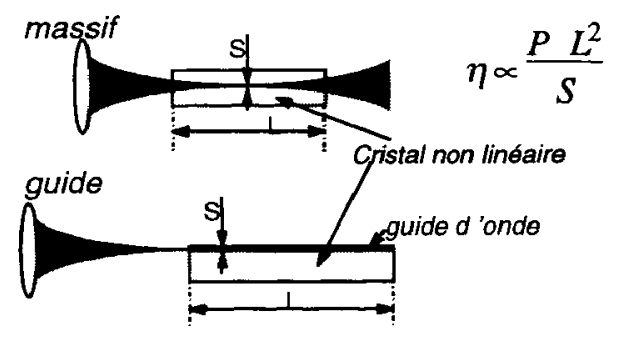

Figure 3.1 : Comparaison entre les configurations massive et guidée.

dans la configuration confocale définie par $2 \mathrm{~L}=\mathrm{Sn} / \lambda$ [31]. Dans un guide d'onde, ce couplage entre section minimale et longueur d'interaction n'existe pas. Une fois que la lumière est couplée dans le guide, elle reste confinée sur toute sa longueur qui peut être choisie en fonction du taux de conversion et des pertes à la propagation. 


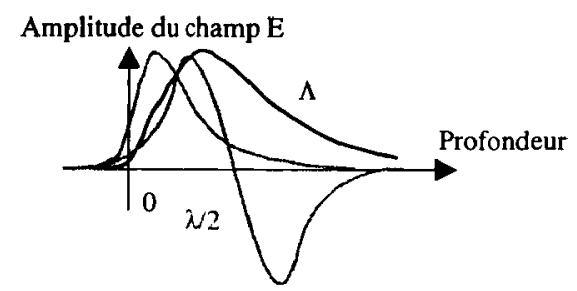

Figure 3.2 : Recouvrement entre les différents modes

La figure 3.2 illustre le problème du recouvrement dans le cas de la génération d'harmonique. Si le guide d'onde est monomode à la fréquence fondamentale, il est multimode à la fréquence harmonique. Les profils de champ correspondants sont tracés dans la figure 3.2 et l'intégrale de recouvrement $I_{R}$ est donnée par :

$$
I_{R} \propto \frac{\left(\iint_{S} \mathbf{d}(x, y) \cdot \mathbf{E}_{\omega}^{2}(x, y) \cdot \mathbf{E}_{2 \omega}(x, y) d x d y\right)^{2}}{\left(\iint_{S} \mathbf{E}_{\omega}^{2}(x, y) d x d y\right)^{2} \iint_{S} \mathbf{E}_{2 \omega}(x, y) d x d y}
$$

On voit donc que si l'harmonique est créé dans le mode d'ordre un, le changement de signe du champ associé fait que le recouvrement est très faible si la non-linéarité est constante

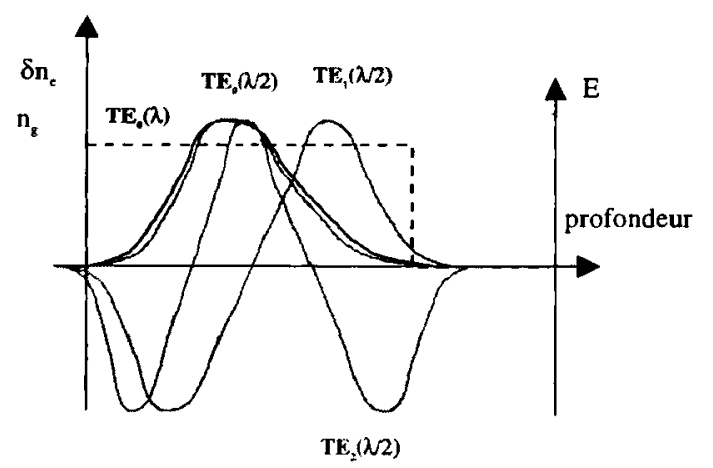

Figure 3.3 : Profil d'indice (en pointillé) et amplitudes des champs associés à la fréquence fondamentale (en noir) et à la fréquence harmonique (en gris) dans un guide à saut d'indice.

sur toute la section du guide. Le taux de conversion, qui est proportionnel à $I_{R}$, sera également très faible. Ceci est particulièrement vrai dans les guides à saut d'indice, où la forme des modes est presque indépendante de la fréquence. Le recouvrement entre les modes d'ordre zéro est donc beaucoup plus important que tous les autres (figure 3.3).

Ceci ne veut cependant pas dire que l'interaction entre les modes fondamentaux est toujours la plus efficace. En effet, la plupart des guides utilisés ont des profils à gradient d'indice, et dans ce cas, le mode d'ordre zéro à la fréquence fondamentale est très différent du mode d'ordre zéro à la fréquence harmonique (figure 3.4). Il est alors nécessaire de pouvoir disposer d'autres paramètres pour ajuster ce profil d'indice. Ceci peut être fait en déposant une couche linéaire de haut indice à la surface du guide et en ajustant son épaisseur pour que le premier lobe du mode harmonique y soit confiné (figure 3.5) permettant ainsi un bon recouvrement dans la partie non linéaire entre son second lobe et le mode fondamental à la fréquence de pompe [32]. 


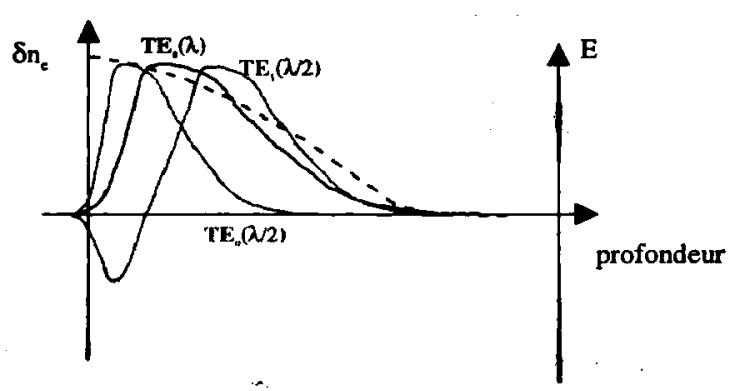

Figure 3.4 : Profil d'indice (en pointillé) et amplitudes des champs associés à la fréquence fondamentale (en noir) et à la fréquence harmonique (en gris) dans un guide à gradient d'indice.

Dans la suite de ce chapitre, nous allons maintenant nous concentrer sur les différentes configurations qui ont permis d'obtenir des rendements de conversion importants, en détaillant chaque fois les techniques utilisées pour réaliser le composant.

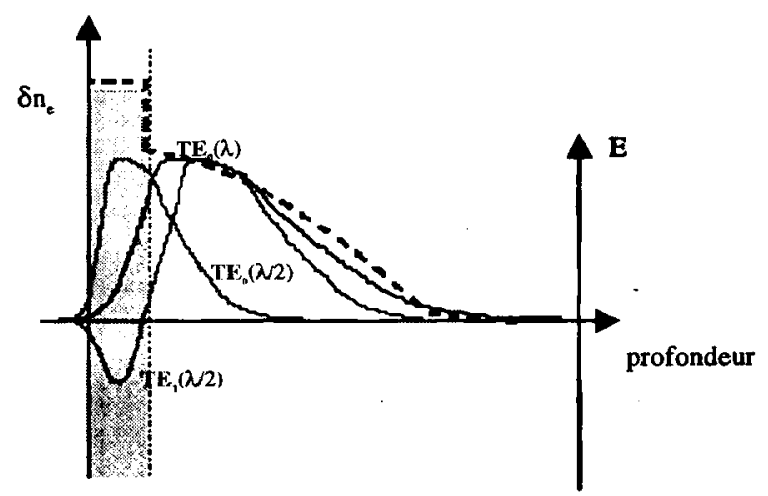

Figure 3.5 : Profil d'indice (en pointillé) et amplitude des champs associés à la fréquence fondamentale (en noir) et à la fréquence harmonique (en gris) dans un guide à gradient d'indice recouvert d'une couche linéaire (grisée)

\subsection{Accord de phase parfait dans les guides d'onde}

Pour déterminer rapidement les possibilités d'accord de phase d'un composant, il est utile de tracer un diagramme d'accord de phase [33] que nous définirons en nous plaçant dans le cas de la génération d'harmonique en type I. Si l'on utilise la biréfringence du matériau pour compenser la dispersion chromatique, on peut trouver la longueur d'onde d'accord de phase en traçant $n_{i}(\lambda)$ et $n_{j}(\lambda / 2)$ en fonction de $\lambda$, i et $j$ représentant deux directions de polarisation. Le point d'accord de phase est donné par le point d'intersection entre les deux courbes (figure 3.6).

Dans le cas des guides d'onde, la situation devient plus complexe, puisque plusieurs modes peuvent exister aux différentes fréquences. Il faut alors calculer et tracer toutes les courbes $n_{\text {eff }}^{k}(\lambda)$ et $n_{\text {eff }}^{\prime}(\lambda / 2)$ et répertorier tous les points d'intersection (figure 3.7).

Ceci peut être simplifié en remarquant que l'indice effectif d'un mode est toujours compris entre l'indice du substrat et l'indice maximum de la couche guidante, qui, dans la 


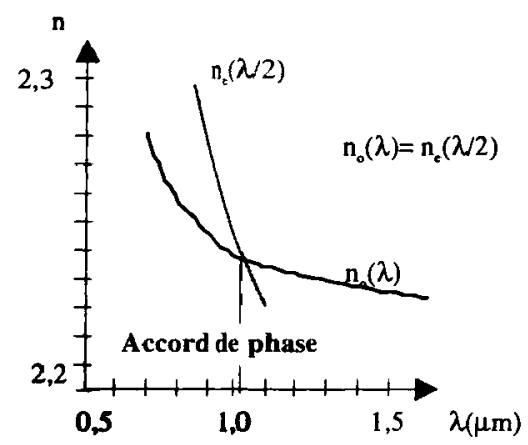

Figure 3.6 : Diagrame d'accord de phase dans un matériau biréfringent.

majeure partie des cas, est l'indice de surface. Il suffit alors de tracer $n_{\text {sub }}(\lambda), n_{\text {surr }}(\lambda), n_{\text {sub }}(\lambda / 2)$ et $\mathrm{n}_{\text {surf }}(\lambda / 2)$. La surface délimitée par ces quatre courbes définit la plage d'accord de phase du guide considéré (figure 3.8 ).

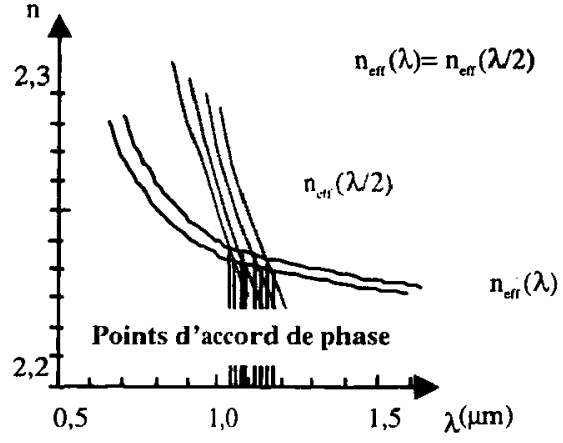

Figure 3.7 : Diagrame d'accord de phase d'un guide d'onde.

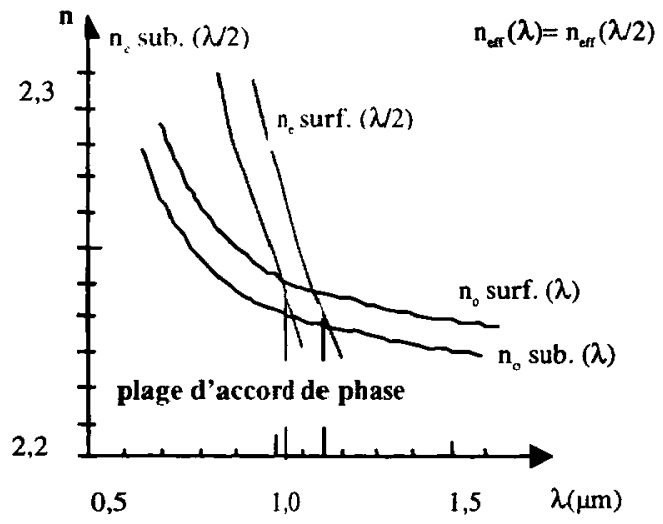

Figure 3.8 : Diagramme d'accord de phase simplifié d' un guide d'onde. 


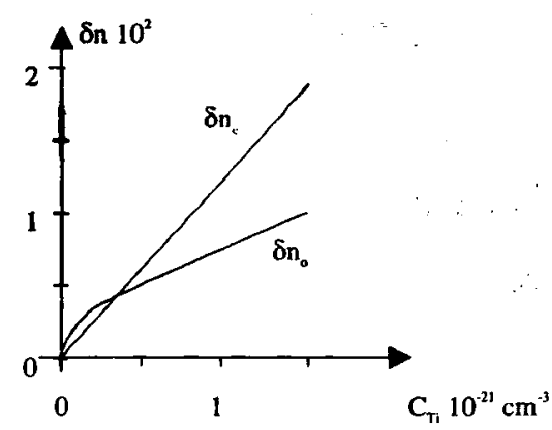

Figure 3.9 : Variation des indices ordinaire et extraordinaire en fonction de la concentration de titane.

\subsubsection{Accord de phase par biréfringence}

Les guides fabriqués par diffusion de Ti dans le niobate de lithium [34] ont une plage d'accord de phase située autour de $1,1 \mu \mathrm{m}$ (figure 3.8). Elle est obtenue en utilisant la biréfringence négative, une onde fondamentale ordinaire et une onde harmonique extraordinaire couplées à l'aide du coefficient non linéaire $d_{31}\left(d_{31}=5 \mathrm{pm} / \mathrm{V}\right)$. La position exacte de l'accord de phase dépend de la géométrie du guide. Dans la figure 3.8, on voit que dans les guides diffusés titane où l'augmentation d'indice ne dépasse jamais 0,02 , la plage d'accord de phase est très limitée autour de $1,1 \mu \mathrm{m}$. La dépendance en température des indices ne permet qu'un déplacement très réduit de cette plage.

Cette configuration a été utilisée par plusieurs auteurs [35] qui ont soigneusement étudié l'évolution de $\delta n_{\circ}$ et $\delta n_{c}$ en fonction de la concentration de Ti (figure 3.9) afin de pouvoir optimiser le recouvrement entre modes fondamentaux aux deux longueurs d'onde. En effet, en

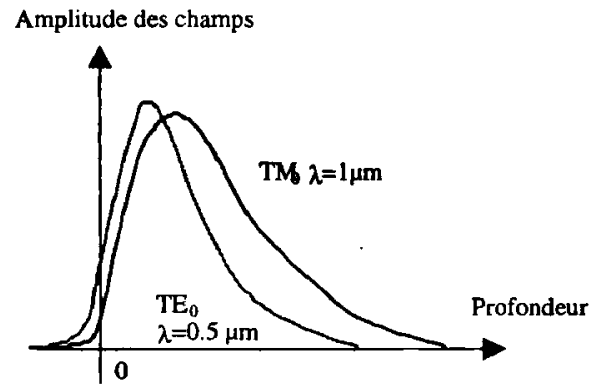

Figure 3.10 : Profil des modes dans un guide réalisé par diffusion de Ti et optimisé pour la génération d'harmonique autour de $1 \mu \mathrm{m}$.

utilisant le fait que la variation de l'indice ordinaire est une fonction non linéaire de la concentration de $\mathrm{Ti}$ et que pour les faibles concentrations $\delta n_{o}$ est plus grand que $\delta n_{c}$ il est possible d'accorder les formes des modes en interaction (figure 3.10).

En utilisant un résonateur [36] pour augmenter encore la densité de puissance, il leur a été possible d'atteindre des efficacités de conversion atteignant les $100 \% / \mathrm{W}$, mais la puissance harmonique était limitée à quelques $\mathrm{mW}$ en raison de l'effet photoréfractif, présent malgré une température d'accord de phase de $250^{\circ} \mathrm{C}$. 


\subsubsection{Accord de phase par dispersion modale}
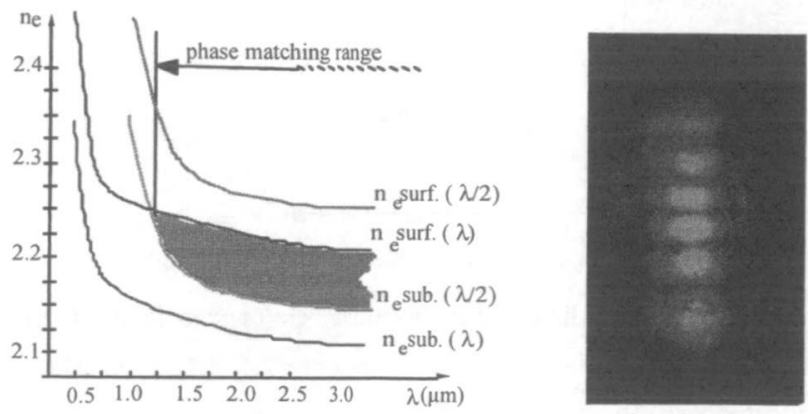

Figure 3.11 : Plage d'accord de phase d'un guide PE présentant une forte augmentation d'indice extraordinaire et champ proche du mode harmonique dans le cas où l'accord de phase est obtenu avec le mode d'ordre 5.

Deux types d'essais ont également été faits pour utiliser le meilleur coefficient du niobate de lithium, $d_{33}=30 \mathrm{pm} / \mathrm{V}$, et pour éviter les limitations dues à l'effet photoréfractif en utilisant la forte augmentation d'indice $\left(\delta n_{c}=0,1\right)$ et la bonne tenue en puissance des guides réalisés par échange protonique. Le premier type d'essais a consisté à utiliser la forte dispersion modale due au $\delta n_{c}$ important, pour compenser la dispersion chromatique. La figure 3.11, montre que cela conduit à une plage d'accord de phase très étendue, mais cette configuration souffre d'une efficacité réduite par un mauvais recouvrement. En effet, la condition d'accord de phase est satisfaite entre un mode d'ordre peu élevé à la fréquence fondamentale et un mode d'ordre élevé à la fréquence harmonique. Une photo de la distribution d'énergie dans le mode harmonique est donnée sur la figure 3.11, et cela montre clairement que, dans ce cas l'intégrale de recouvrement et par conséquent l'efficacité de conversion, sont très réduites [37].
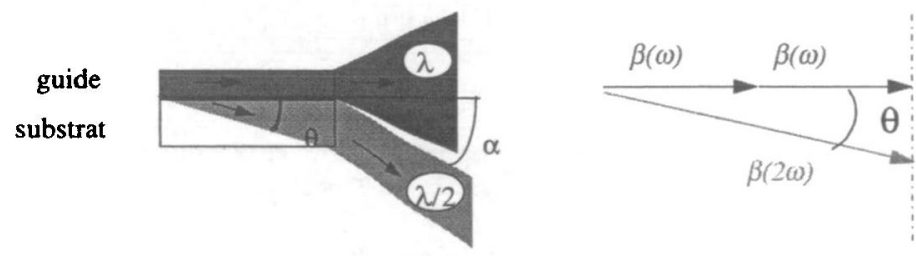

Figure 3.12 : Configuration Cerenkov.

Le deuxième type d'essais a consisté à utiliser les modes de rayonnement dans la configuration dite "Cerenkov". Dans ce cas la polarisation à la fréquence harmonique $2 \omega$ a une vitesse de phase supérieure à celle d'une onde guidée à $2 \omega$. Cette polarisation est donc la source d'une onde rayonnée à $2 \omega$, qui se propage dans le substrat avec un angle $\theta$ par rapport au guide (figure 3.12 ). Cet angle $\theta$ satisfait automatiquement la condition d'accord de phase 
$\mathrm{n}_{\text {eff }}(\omega)=\mathrm{n}(2 \omega) \cos \theta$, ce qui permet encore une fois d'avoir une plage d'accord de phase très étendue. Une conception adaptée du guide [38], ou une combinaison avec les techniques de quasi-accord de phase [39], permet de garder $\theta$ petit ce qui maximise le recouvrement et la longueur d'interaction. Les meilleurs résultats obtenus dans cette configuration sont rapportés par K. Yamamoto [40], qui a obtenu $1,6 \mathrm{~mW}$ dans le bleu, à partir de $100 \mathrm{~mW}$ d'infrarouge couplé dans le guide. Bien que la distribution du champ harmonique soit quelque peu inusuelle, cette configuration peut s'avérer utile pour certaines applications en raison de ses propriétés d'accord de phase automatique.

\subsection{Quasi-accord de phase dans les guides d'onde}

Le quasi-accord de phase 2 est de nos jours, une des configurations les plus utilisées pour les interactions à trois ondes dans les matériaux massifs. Historiquement, elle a d'abord été utilisée en optique intégrée, configuration qui permet de n'inverser que de très petits volumes.

De plus, les techniques d'inversion périodique basées sur un gradient de composition et un traitement thermique peuvent également créer le guide d'onde [41]. Le principal avantage du quasi-accord de phase est qu'en ajustant la périodicité, il permet d'obtenir, à température ambiante, une interaction efficace entre n'importe quel triplet de longueur d'onde situé dans la plage de transparence du matériau et vérifiant la relation de conservation de l'énergie.

Cette configuration est particulièrement intéressante dans les matériaux ferroélectriques où la périodicité du $\chi^{(2)}$ est obtenue en inversant périodiquement la polarisation du cristal. Depuis 1993, on a mené de nombreuses études pour parvenir à contrôler ce processus et aujourd'hui de nombreuses techniques ont été adaptées au $\mathrm{LiNbO}_{3}$, au $\mathrm{LiTaO}_{3}$, au KTP et au RTA [42, 43, 44, 45, 46, 47, 48]. Dans cette configuration, les guides d'onde introduisent les mêmes avantages et les mêmes exigences supplémentaires que dans le cas de l'accord de phase parfait, et les résultats obtenus en fluorescence paramétrique vont nous permettre d'illustrer leurs possibilités.

\subsubsection{Influence des paramètres du guide}

La fluorescence paramétrique est obtenue en injectant seulement le faisceau pompe dans le guide. Si sa longueur d'onde est adaptée, elle se couple non linéairement avec les fluctuations du vide, et l'on observe deux ondes de fréquence plus basse, appelées signal et complémentaire (idler) qui vérifient les relations de conservation de l'énergie et de l'impulsion (accord de phase). Comme l'accord de phase parfait, le quasi-accord de phase peut être obtenu entre des modes guidés uniquement ou faire intervenir des modes rayonnés. La figure 3.13 présente un moyen de résoudre graphiquement les équations de conservation dans le cas où la pompe et le signal sont guidés, et où la plus grande longueur d'onde, l'idler est soit guidée soit rayonnée. Quand tous les modes sont guidés, il est possible de tracer une courbe d'accord de phase où les couples signal et idler sont reportés en fonction de la longueur d'onde de pompe.

Les simulations numériques montrent que bien que les modifications d'indice introduites par la fabrication des guides soient généralement faibles (1 à $2 \%$ ), leur influence sur les courbes d'accord de phase est très importante (figure 3.14). Une analyse détaillée révèle qu'il en est de même pour tous les autres paramètres permettant de définir le profil d'indice, sa forme (figure 3.15), sa profondeur (figure 3.16) et sa largeur (figure 3.17). Cette sensibilité est tellement grande que la détermination expérimentale de la courbe d'accord de phase est un moyen extrêmement précis de déterminer le profil d'indice du guide. 


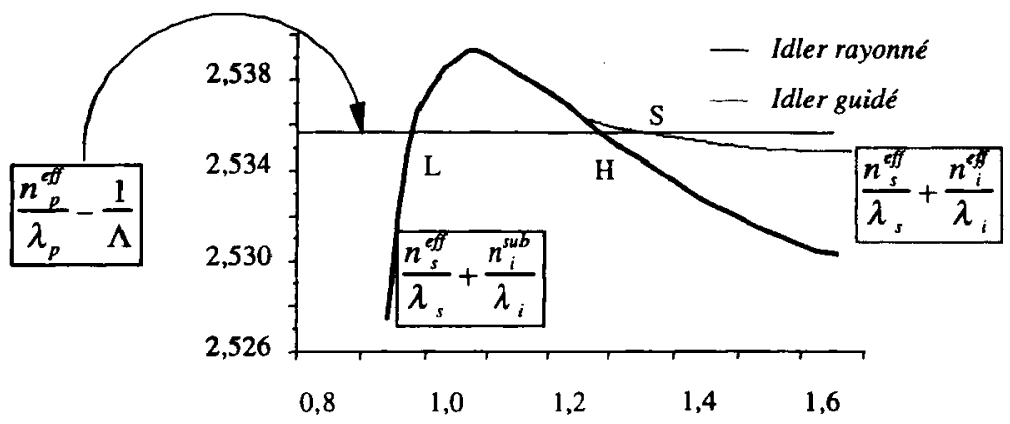

Figure 3.13 : Détermination géométrique de la longueur d'onde signal dans une interaction paramétrique en configuration de quasi-accord de phase. La courbe "idler rayonné" correspond au cas limite où l'angle de radiation $\theta=0$.

\subsubsection{Observation expérimentale de la fluorescence paramétrique}

Actuellement, les générateurs paramétriques les plus efficaces sont constitués par des guides d'ondes réalisés dans du niobate de lithium polarisé périodiquement. La polarisation est obtenue soit par diffusion de titane soit par application d'un champ électrique, et les guides d'ondes fabriqués ensuite en utilisant la technique d'échange protonique. En injectant quelques $\mathrm{mW}$ de l'onde pompe au voisinage de $800 \mathrm{~nm}$ dans ces guides, on obtient en sortie deux ondes signal et idler avec une efficacité de conversion qui varie entre $10^{-10}$ et $10^{-6}$ suivant la qualité du guide d'onde. Bien que faible, cette fluorescence peut être très précisément analysée.

Pour cela on image le champ proche en sortie du guide sur la fente d'entrée d'un monochromateur, après avoir filtré la pompe. En sortie du monochromateur, on dispose d'un détecteur Germanium refroidi à l'azote qui permet de mesurer le signal jusqu'à $1,7 \mu \mathrm{m}$. La figure 3.18 donne un exemple de spectre recueilli à la sortie d'un guide de $8 \mathrm{~mm}$ de long réalisé sur un substrat de PPLN présentant une période d'inversion de $18 \mu \mathrm{m}$.

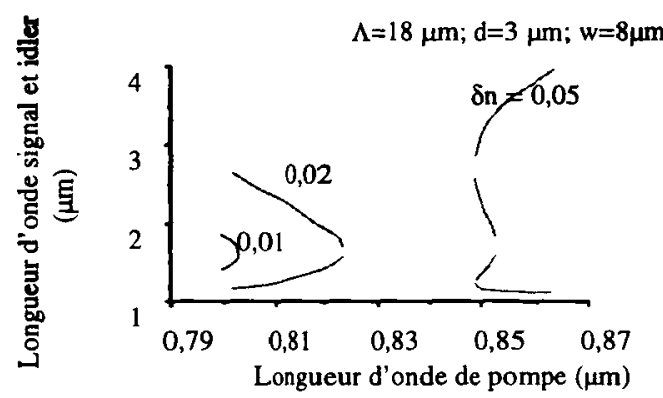

Figure 3.14 : Influence de l'augmentation d'indice sur la courbe d'accord de phase. 


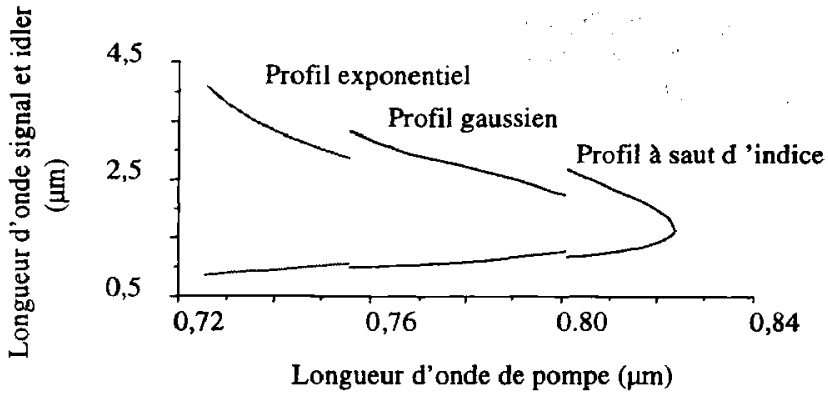

Figure 3.15 : Influence de la forme du profil d'indice sur la courbe d'accord de phase.

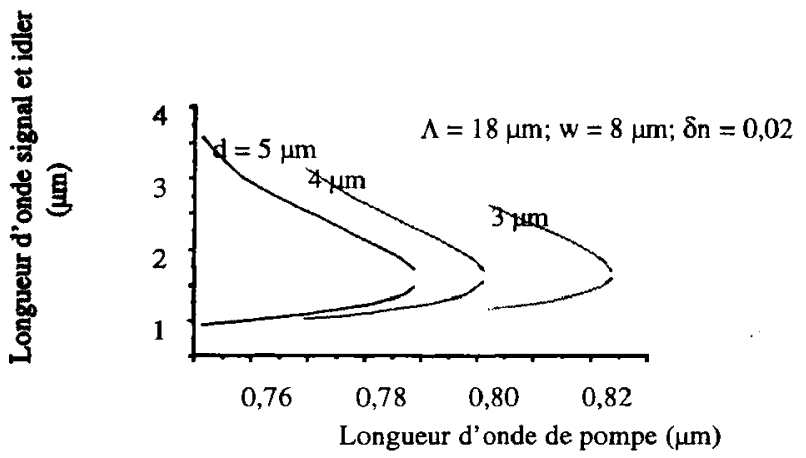

Figure 3.16 : Influence de la profondeur du guide sur la courbe d'accord de phase.

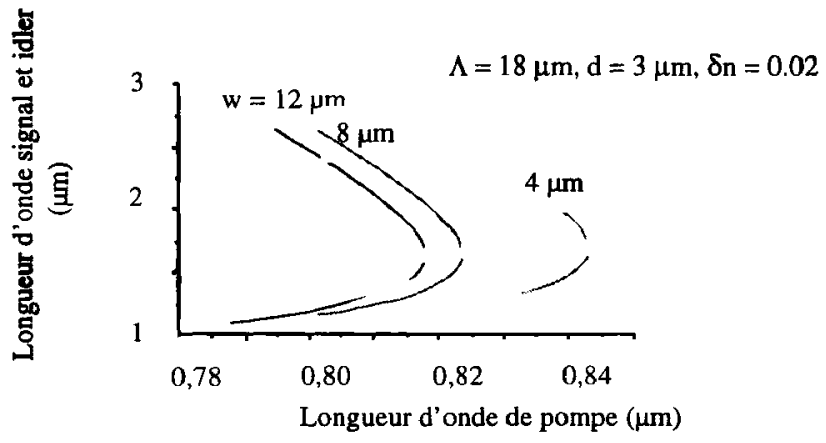

Figure 3.17 : Influence de la largeur du guide sur la courbe d'accord de phase. 
Ce spectre présente un pic bien défini qui correspond au signal obtenu par interaction entre modes guidés (le pic correspondant à l'idler ne peut pas être vu car il est au-delà de la plage de détection du détecteur $\mathrm{Ge}$ ). Il présente également à des fréquences plus élevées, un large continuum qui correspond au signal guidé associé à un idler rayonné. Du côté des courtes longueurs d'ondes, ce continuum est limité par le filtre de réjection de la pompe [49].

En faisant varier la longueur d'onde de pompe et en relevant la longueur d'onde correspondant au pic de fluorescence, on peut tracer la courbe d'accord de phase expérimentale correspondant à l'échantillon étudié. La figure 3.19 montre le déplacement de cette courbe d'accord de phase en fonction de la période d'inversion des domaines, et elle permet de constater que pour une variation de $15 \mathrm{~nm}$ de la longueur d'onde de pompe, on couvre une plage de plus de $1 \mu \mathrm{m}$ avec le signal et lidler. Comme on a pu le voir numériquement et expérimentalement, le profil d'indice peut modifier très sensiblement les courbes d'accord de phase. Ceci a deux conséquences très importantes d'un point de vue pratique. Tout d'abord en ajustant les courbes d'accord de phase numériques sur les courbes expérimentales, il est possible de déterminer avec une grande précision les paramètres du

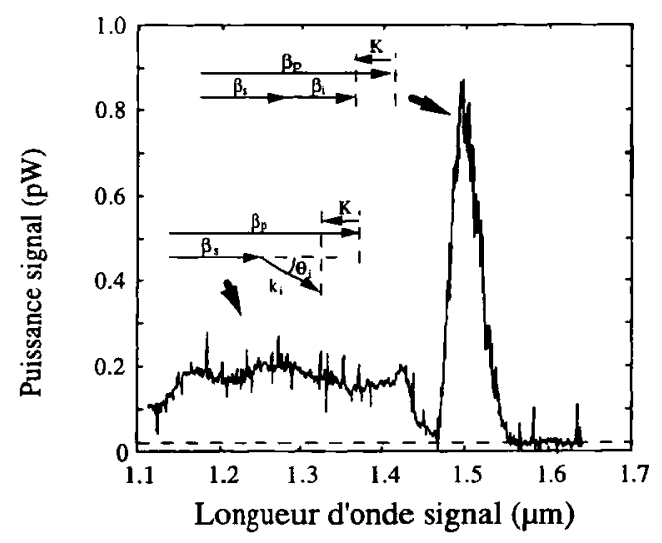

Figure 3.18 : Spectre de fluorescence montrant le pic signal correspondant à l'internaction entre modes guidés et le continuum correspondant à l'association avec un idler rayonné.

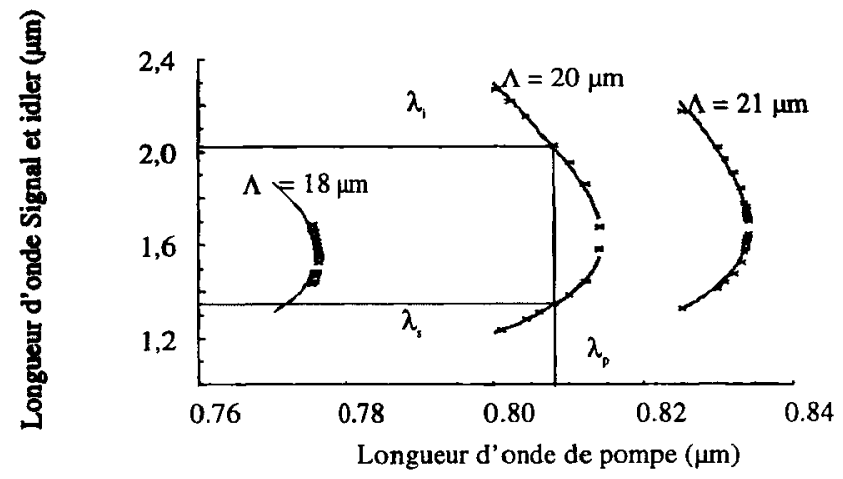

Figure 3.19 : Influence de la période de polarisation sur les courbes d'accord de phase. Les croix correspondent à des points expérimentaux et les lignes à des calculs numériques utilisant les mêmes paramètres pour le guide. Seule $\Lambda$ est changée. 
guide $\left(\delta n_{c} \pm 0,0001\right.$, largeur et profondeur $\left.\pm 0,1 \mu \mathrm{m}\right)$. D'autre part l'extrême sensibilité aux modifications d'indice montre qu'en optique intégrée, il est possible d'envisager plusieurs processus d'ajustement fin de la longueur d'onde de sortie.

Accordabilité. La première possibilité est d'utiliser la température pour ajuster la longueur d'onde de sortie, et la figure 3.20 indique les variations possibles en utilisant une

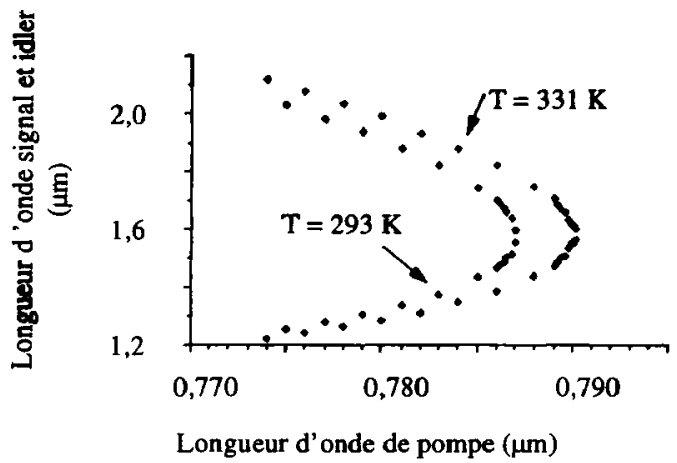

Figure 3.20 : Influence de la température sur la courbe d'accord de phase d'un guide échange protonique recuit, réalisé sur un cristal de niobate de lithium polarisé périodiquement par diffusion de $\mathrm{Ti}$.

augmentation de température de $40^{\circ} \mathrm{C}$. Les processus faisant intervenir la température sont cependant toujours lents, et le principal intérêt de chauffer les guides est de réduire très sensiblement les problèmes d'effet photoréfractif. La configuration la plus intéressante serait sans doute de profiter de la structure planaire pour utiliser l'effet électro-optique pour ajuster les longueurs d'onde signal et idler ou pour les stabiliser [50]. La figure 3.21 illustre cette possibilité, en montrant qu'une variation d'indice de $\pm 0,0005$ permet de déplacer le signal de $\pm 50 \mathrm{~nm}$ et l'idler de $\pm 100 \mathrm{~nm}$.

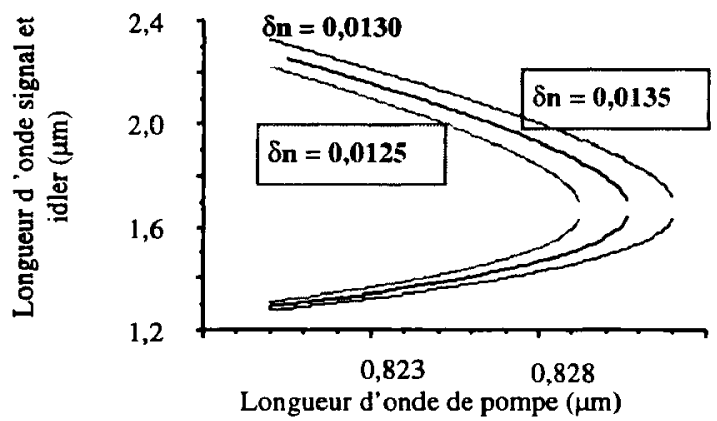

Figure 3.21 : Influence de l'effet électro-optique sur les courbes d'accord de phase.

Effet photoréfractif. À température ambiante, dans un guide recuit de $7 \mu \mathrm{m}$ de large $(\Lambda=20 \mu \mathrm{m})$ on a pu recueillir avec une pompe faible $(<1 \mathrm{~mW})$ une courbe d'accord de phase qui correspond à celle que l'on obtient numériquement en utilisant des profils d'indice exponentiels en profondeur et en largeur, $\delta \mathrm{n}=0,0143, \mathrm{~d}=2,1 \mu \mathrm{m}$ et $w=3,5 \mu \mathrm{m}$. En augmentant la puissance de pompe jusqu'à $20 \mathrm{~mW}$, on déplace la courbe d'accord de phase de $5 \mathrm{~nm}$ vers les courtes longueurs d'onde (figure 3.22). 
Le même déplacement peut être obtenu numériquement en réduisant l'augmentation d'indice de $1,8 \times 10^{-3}$, ce qui peut être obtenu par effet photoréfractif [51]. La plupart du temps, l'effet photoréfractif est considéré comme une perturbation puisqu'il introduit des instabilités

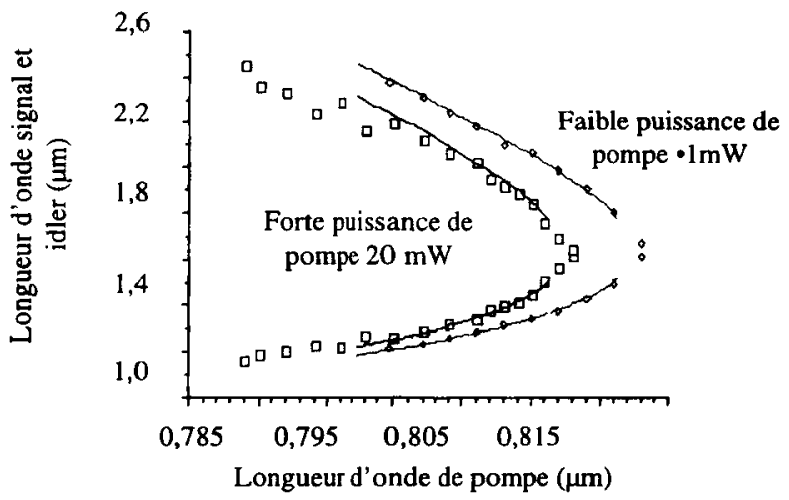

Figure 3.22 : Influence de l'effet photoréfractif sur la courbe d'accord de phase d'un guide échange protonique recuit réalisé sur un substrat de niobate de lithium polarisé périodiquement par diffusion de $\mathrm{Ti}$.

dans le fonctionnement des composants. Par exemple, pour une longueur d'onde de pompe fixée, on a pu relever un changement important de la longueur d'onde signal $(20 \mathrm{~nm})$ en augmentant la puissance couplée dans le guide de 1 à $30 \mathrm{~mW}$ (figure 3.23).

Ceci est incompatible avec la réalisation de composant du type Oscillateur Paramétrique Optique Intégré (OPOI). Cependant cet effet peut être dramatiquement réduit en chauffant le substrat de quelques dizaines de degrés. Un exemple de ce type de fonctionnement est donné sur la figure 3.23 où l'on voit que quand le substrat est porté à $60^{\circ} \mathrm{C}$, la longueur d'onde signal ne dépend plus de la puissance de pompe couplée dans le guide.

L'efficacité de conversion peut également être perturbée par l'effet photoréfractif. En effet, si les indices effectifs dépendent de la puissance couplée, il en est de même des

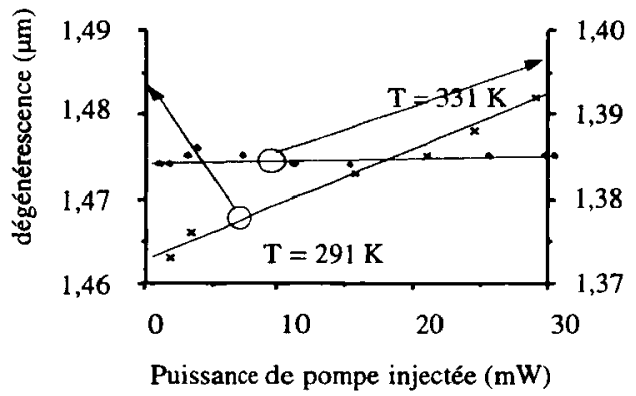

Figure 3.23 : Suppression de l'effet photoréfractif en chauffant le substrat d'un guide échange protonique recuit réalisé sur un substrat de niobate de lithium polarisé périodiquement par diffusion de Ti.

distributions des champs et donc de l'intégrale de recouvrement. Ceci peut être observé en traçant la puissance de signal en fonction de la puissance de pompe couplée, et l'on a pu observer qu'à température ambiante et au-delà de quelques dizaines de $\mathrm{mW}$ injectés, la puissance signal sature contrairement à la théorie qui prévoit une dépendance linéaire. Cette dépendance peut cependant être observée quand le substrat est porté à $60^{\circ} \mathrm{C}$ (figure 3.24 ). 


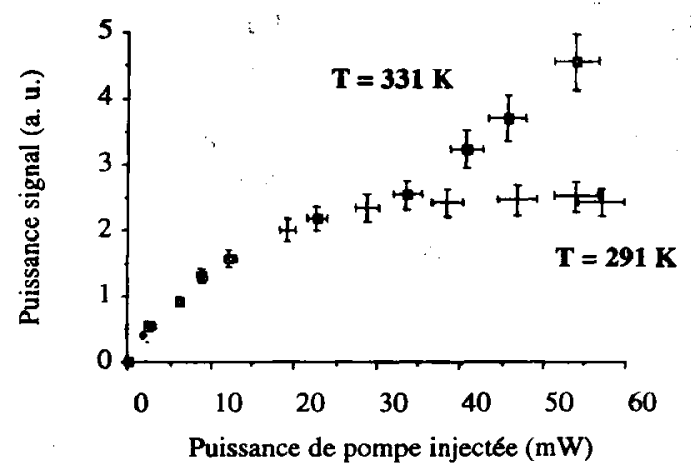

Figure 3.24 : Influence de l'effet photoréfractif et de la température sur la puissance de sortie d'un guide échange protonique recuit sur un substrat de niobate de lithium polarisé périodiquement par diffusion de $T i$.

\subsubsection{Génération de différence de fréquence}

Les mesures effectuées en fluorescence paramétrique permettent de déterminer la longueur d'onde de pompe qu'il faut choisir pour obtenir dans un guide donné et à partir d'un signal fixé, une onde idler créée par différence de fréquence (DFG). On peut alors mesurer les puissances signal et idler en fonction de la puissance de pompe. Pour mesurer, à l'aide d'une détection synchrone, le signal amplifié il faut moduler le signal injecté et la pompe, mais pour

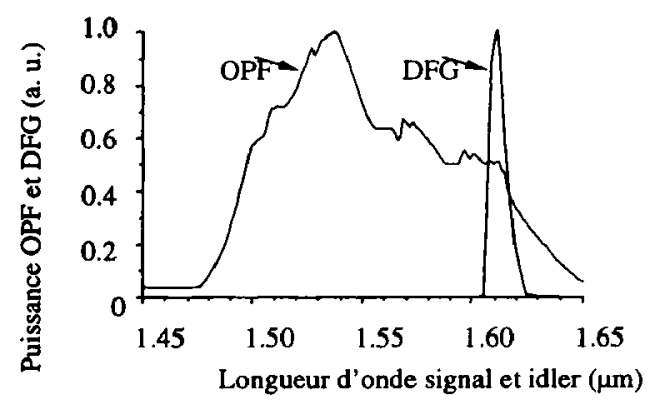

Figure 3.25 : Signal obtenu par différence de fréquence (DFG) comparé au spectre de fluorescence paramétrique(OPF) obtenu dans le même guide

mesurer l'idler créé il vaut mieux ne moduler que la pompe pour éviter les perturbations dues au signal car les deux longueurs d'onde sont proches $(60 \mathrm{~nm})$ mais la puissance idler est trois à quatre ordres de grandeur inférieure à celle du signal. La figure 3.25 montre le pic idler obtenu par DFG, superposé au signal de fluorescence paramétrique obtenu dans le même guide. En dépit de ces possibilités très attrayantes, et du nombre de réalisations, il reste encore un certain travail à faire sur les guides d'ondes réalisés dans les matériaux polarisés périodiquement, puisque tous les composants réalisés montrent des efficacités de conversion qui restent inférieures aux prédictions théoriques. Pour comprendre cette difficulté il faut entrer dans le détail des processus de fabrication, et comme la majorité des composants réalisés à ce jour sont basés sur l'échange protonique dans le PPLN ou PPLT, nous nous concentrerons sur ces deux matériaux. 


\section{FABRICATION DES GUIDES DANS LE NIOBATE ET LE TANTALATE DE LITHIUM POLARISÉ PÉRIODIQUEMENT.}

En optique non linéaire, ces deux cristaux occupent une place toute particulière et sont considérés comme une référence par de nombreux auteurs. En effet, la plupart des composants qui ont été réalisés reposent sur l'un deux. Pour apprécier l'état de l'art en optique intégrée non linéaire, il est donc important de connaître les avantages et les inconvénients des différentes techniques disponibles pour réaliser des guides d'ondes dans ces cristaux.

Sur niobate de lithium, la plus fréquemment utilisée est la diffusion de Ti qui permet d'augmenter l'indice ordinaire et l'indice extraordinaire. Cette technique nécessite cependant de chauffer le cristal au voisinage (pour $\mathrm{LiNbO}_{3}$ ) et au-dessus (pour $\mathrm{LiTaO}_{3}$ ) de sa température de Curie, ce qui la rend difficilement compatible avec la polarisation périodique. C'est pour cette raison que la plupart des tentatives visant à obtenir des guides dans PPLN ou PPLT on fait appel à l'échange protonique pour créer les guides d'onde dans des substrats préalablement polarisés périodiquement.

Evidemment, en utilisant la technique de polarisation par champ électrique on pourrait procéder dans l'autre sens, en tentant de polariser le cristal après avoir créé les guides d'onde. L'influence des différents types d'échange protonique sur la réponse à la polarisation du $\mathrm{LiNbO}_{3}$ a été récemment mentionnée [52, 53], mais étant donné la complexité de l'échange protonique et le nombre de modifications que cet échange [54] peut introduire dans le cristal, cette étude n'en est qu'au tout début.

\subsection{Diagramme de phase des couches échangées}

En utilisant un large éventail de paramètres de fabrication et plusieurs orientations cristallines, nous avons pu identifier 5 phases cristallines pour le composé $\mathrm{H}_{\mathrm{x}} \mathrm{Li}_{1 \cdot \mathrm{x}} \mathrm{TaO}_{3}$ et 7 pour le composé $\mathrm{H}_{\mathrm{x}} \mathrm{Li}_{1-\mathrm{x}} \mathrm{NbO}_{3}$. Les échanges étaient réalisés sur des substrats de qualité optique dont

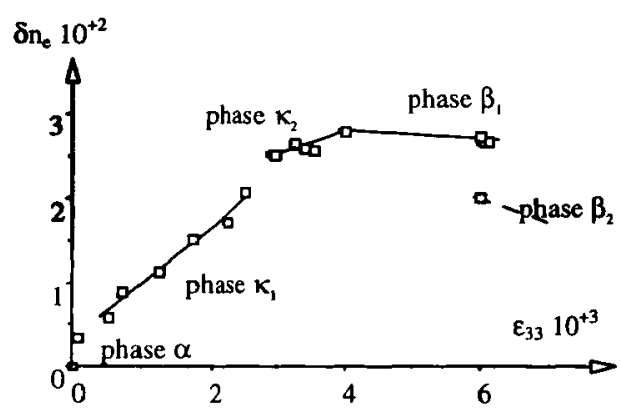

Figure 4.1 : Diagrame de phase d'une couche de $\mathrm{H}_{\mathrm{x}} \mathrm{Li}_{1-\mathrm{x}} \mathrm{TaO}_{3}$ à la surface d'un cristal orienté $\mathrm{Z \perp}$

la surface était perpendiculaire soit à l'axe $X(X \perp)$ soit à l'axe $Z(Z \perp)$, en utilisant comme source de protons des bains de différentes acidités. Certains échantillons ont également été recuits entre $320^{\circ}$ et $400^{\circ} \mathrm{C}$ pour redistribuer les protons.

Les guides plans ainsi obtenus ont été caractérisés par la technique du coupleur à prisme qui permet de mesurer les indices effectifs des modes à partir desquels les profils d'indice sont reconstitués en utilisant la technique IWKB [55]. Nous avons ensuite reconstitué la structure cristalline à la surface de ces mêmes échantillons en utilisant la technique des rayons $X$ rasants pour mesurer les distances entre différents plans cristallins [56]. 
Les diagrammes de phase, présentés sur les figure 4.1 et 4.2 , sont le résultat de la compilation des résultats obtenus. Dans ces diagrammes, les transitions de phase sont

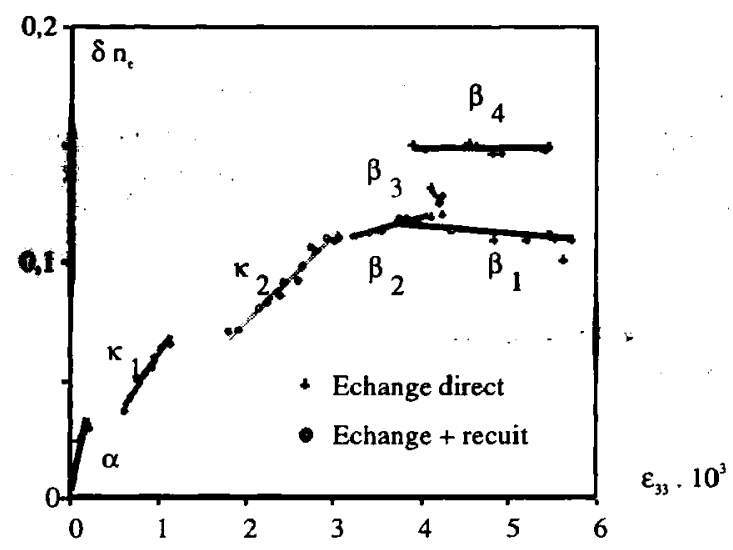

Figure 4.2 : Diagrame de phase d'une couche de $\mathrm{H}_{x} \mathrm{Li}_{1-x} \mathrm{NbO}_{3}$ à la surface d'un cristal orienté $\mathrm{Z \perp}$

caractérisées par des discontinuités qui affectent les variations d'indice $\left(\delta n_{e}\right)$. Cependant, certaines transitions comme la transition $\beta_{1}-\beta_{2}$ sur les substrats de $\mathrm{LiNbO}_{3} \mathrm{Z} \perp$ ne peuvent être mises en évidence qu'en étudiant les déplacements dans les plans perpendiculaires à la surface et en traçant par exemple $\varepsilon_{23}$.

Ces diagrammes de phase montrent que si dans chaque phase la variation d'indice varie

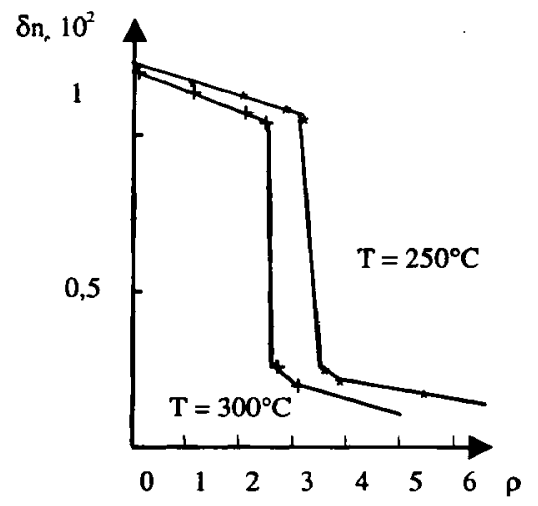

Figure 4.3 : Influence de la température du bain et de sa teneur $\rho$ en benzoate de lithium sur la variation d'indice maximale induite par l'échange protonique.

linéairement avec la déformation qui est supposée proportionnelle à la concentration de protons, le coefficient de proportionnalité varie de manière très importante d'une phase à l'autre. De plus, ils nous ont permis d'expliquer les propriétés optiques des guides produits en utilisant différents types d'échanges protoniques [57,37]. 


\subsection{1 Échange direct}

Les phases $\alpha$ et $\beta$ sont obtenues par échange direct en utilisant des bains d'acidités différentes. Cette acidité peut être choisie en utilisant un bain d'acide benzö̈que contenant un pourcentage massique $\rho$ de benzoate de lithium. Les phases $\beta$ sont obtenues avec l'acide pur ou contenant peu de sel de lithium. En augmentant cette concentration au-delà d'un seuil $\rho_{\mathrm{s}}$ qui dépend de la température du bain, on passe brutalement à la phase $\alpha$ (figure 4. 3).

\subsubsection{Recuit}

Les effets des recuits sur les guides protons peuvent être assez complexes, puisqu'ils peuvent provoquer aussi bien une diminution qu'une augmentation d'indice. Ce comportement compliqué peut cependant être interprété en regardant les diagrammes de phase et en admettant qu'un recuit provoque toujours une réduction de la concentration en proton et donc une diminution de la contrainte $\varepsilon_{33}$. Cependant, durant le recuit, le composé $\mathrm{H}_{x} \mathrm{Li}_{1-x} \mathrm{NbO}_{3}$ ou $\mathrm{H}_{\mathrm{x}} \mathrm{Li}_{1-\mathrm{x}} \mathrm{TaO}_{3}$ peut soit évoluer au sein d'une même phase, soit connaître plusieurs transitions de phases. L'évolution du profil d'indice peut donc être assez compliquée mais parfaitement prédite si l'on connaît exactement la phase de la couche de départ. Par exemple, si l'échange initial a permis d'obtenir une couche de $\mathrm{H}_{x} \mathrm{Li}_{1-\mathrm{x}} \mathrm{TaO}_{3}$ en phase $\beta_{2}$, l'augmentation d'indice $\delta n_{0}$ et la profondeur du guide vont augmenter ce qui correspond à une évolution en phase $\beta_{2}$ suivie d'une transition en phase $\beta_{1}$ ou $\kappa_{2}$ (figure 4 . 1). En continuant le recuit on obtiendra alors une augmentation de profondeur accompagnée d'une réduction du $\delta n_{e}$, ce qui correspond à l'évolution au travers des phases $\kappa_{2}, \kappa_{1}$ et $\alpha$.

Le recuit est la seule façon d'obtenir des couches de type $\kappa$, mais elles ne sont pas très intéressantes puisque qu'elles présentent des pertes à la propagation importantes qui peuvent être dues soit à une mauvaise qualité cristalline soit à une interface perturbée.

\subsection{Absorption infrarouge}

Une autre caractéristique importante des couches échangées peut être étudiée par absorption IR. En effet, après l'échange, les couches ubtenues sur les substrats $X \perp$, présentent une bande d'absorption importante au voisinage de $3 \mu \mathrm{m}$, associée aux vibrations de la liaison $\mathrm{OH}$ [58].

Transmission

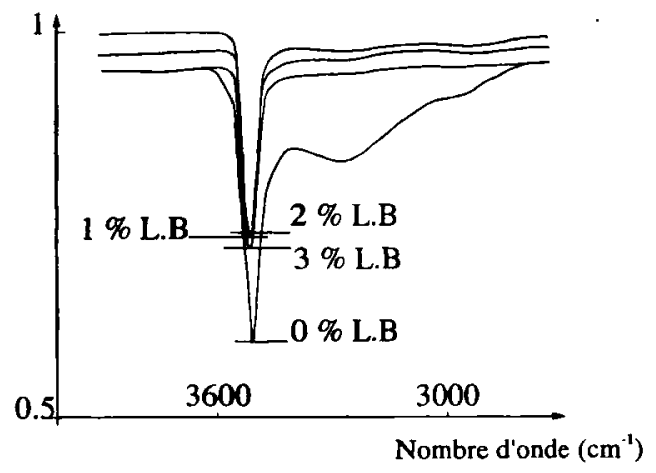

Figure 4.4 : Spectres d'absorption IR des liaisons $\mathrm{OH}$ présentes dans des guides réalisés a $250^{\circ} \mathrm{C}$ avec des bains plus ou moins acides 
Suivant les paramètres de fabrication, le spectre présente un pic bien défini centré à $3510 \mathrm{~cm}^{-1}$ accompagné ou non d'une large bande autour de $3280 \mathrm{~cm}^{-1}$ (figure 4.4) qui tend à disparaître lorsqu'on réduit l'acidité du bain ou au cours des recuits. La corrélation précise entre les spectres d'absorption et les diagrammes de phase reste à faire. Cependant nous avons pu observer [59], que dans le cas des guides élaborés en phase $\alpha$, l'absorption est faible (cette phase correspond à une faible concentration en protons) et centrée à une fréquence légèrement différente $\left(3490 \mathrm{~cm}^{-1}\right)$. Bien que très qualitatives, ces observations sont primordiales car elle révèlent l'existence de plusieurs phonons susceptibles de modifier les propriétés du cristal ou des dopants que l'on peut y introduire.

\subsection{Propriétés non linéaires des guides réalisés par échange protonique sur niobate de lithium.}

Les études des effets de l'échange protonique sur les propriétés non linéaires du cristal ont conduit à une certaine controverse. En effet, tous les auteurs s'accordent à trouver que l'échange protonique peut réduire voire détruire le coefficient non linéaire, mais une partie d'entre eux prétend qu'il est possible de restaurer les propriétés du cristal par un recuit [60, 61], l'autre partie observant des dégradations permanentes [62]. Pour clarifier cette situation, nous avons étudié [63] l'influence de l'échange protonique sur le $\chi^{(2)}$ en mesurant le signal de deuxième harmonique réfléchi par la face d'entrée polie d'un guide [64]. Cette étude nous a permis de montrer qu'il est possible de réaliser des guides par échange protonique direct qui ne présentent aucune réduction du coefficient non linéaire. De plus, ces résultats préliminaires combinés avec ceux qui ont été publiés précédemment tendent à indiquer que la possibilité de restaurer les propriétés du cristal par un recuit, dépend des paramètres utilisés pour réaliser le guide initial.

Pour le guide $\mathrm{PE}_{\mathrm{III}}$ dont le profil d'indice est indiqué sur la figure 4. 5, nous avons utilisé

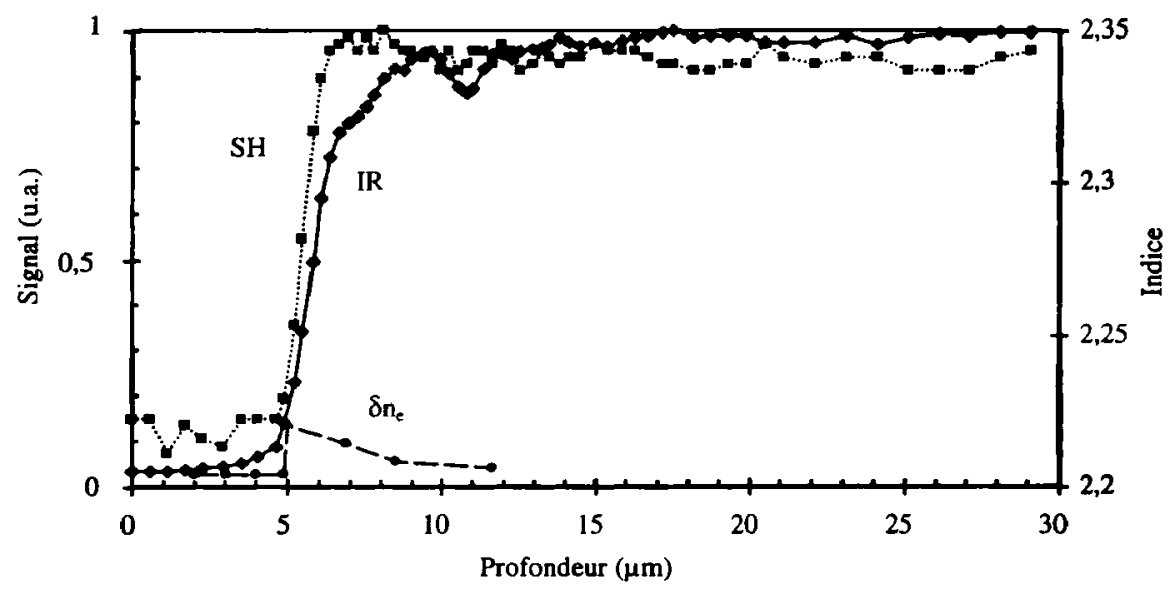

Figure 4.5 : Profil d'indice et signaux (fondamental et deuxième harmonique) réfléchis obtenus sur un guide $\mathrm{PE}_{\mathrm{III}}\left(3 \%\right.$ B.L., $\left.300^{\circ} \mathrm{C}, 70 \mathrm{~h}^{\circ}\right)$

un bain faiblement acide (3\% de benzoate de lithium) pour rester en phase $\alpha$ et un temps d'échange de $70 \mathrm{~h}$ pour obtenir la profondeur d'échange nécessaire. L'enregistrement du signal non linéaire permet de voir qu'il n'y a aucune différence d'intensité ou de qualité de 
faisceau entre la zone échangée et le substrat. Ce type de processus ne perturbe donc pas les propriétés non linéaires du cristal.

Pour un guide $\mathrm{PE}_{\mathrm{t}}$ préparé en utilisant un bain contenant $1 \%$ de benzoate de lithium, (phase $\beta_{1}$ ), le résultat est complètement différent. En effet, en enregistrant le signal de deuxième harmonique obtenu en déplaçant le point de focalisation du fondamental en partant du substrat, on constate que celui-ci disparaît quasiment quand on atteint les couches échangées puisqu'il est inférieur à $5 \%$ du signal mesuré dans le substrat (figure 4.6), ce qui le signe d'un coefficient non linéaire réduit à $20 \%$ de sa valeur dans le massif. À l'interface entre le substrat et la zone échangée, le signal harmonique augmente fortement alors que la qualité du faisceau réfléchi se dégrade sévèrement. Ce fait est encore plus important sur les guides recuits.
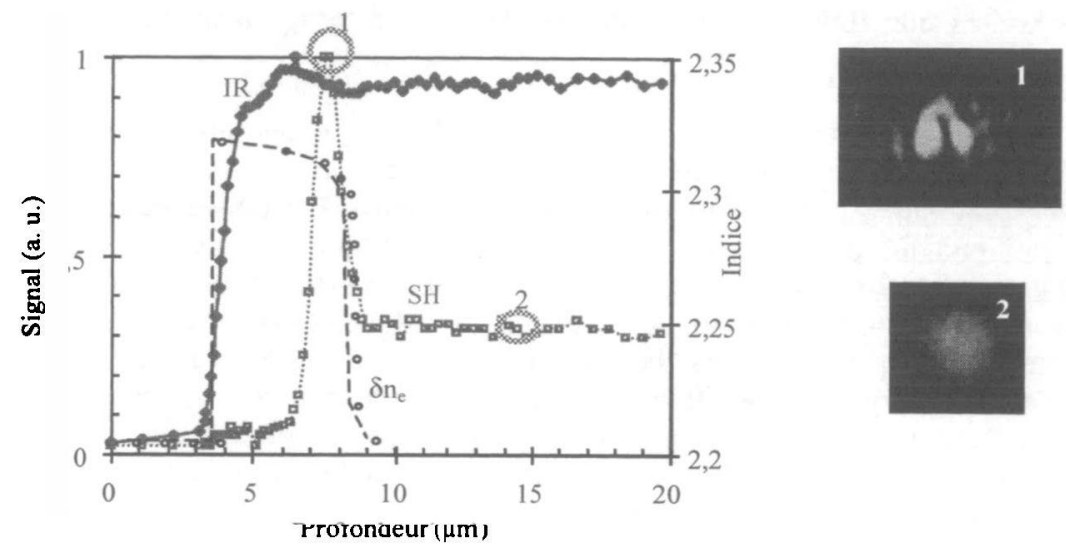

Figure 4.6 : Profil d'indice et signaux (fondamental et deuxième harmonique) réfléchis obtenus sur un guide PE, (1\%B.L., $300^{\circ}, 5$ h). Les photos sur la droite sont celles du faisceau réfléchi prises aux différentes positions repérées par des chiffres.

Sur un des échantillons testés qui a été recuit en plusieurs étapes de traitement à $330^{\circ} \mathrm{C}$ et $350^{\circ} \mathrm{C}$, le signal à l'interface est cent fois plus grand que celui qui provient du substrat

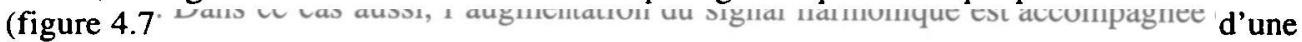
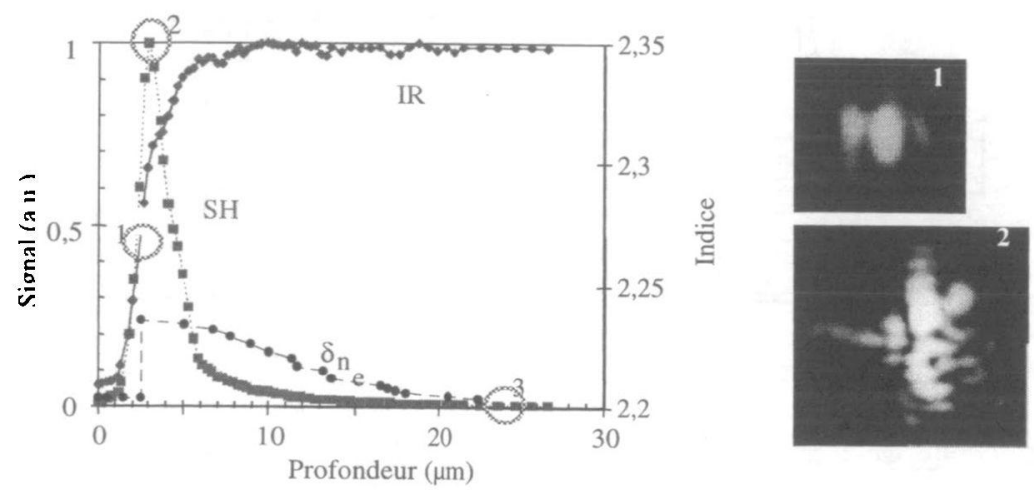

Figure 4.7 : Profil d'indice et signaux (fondamental et deuxième harmonique) réfléchis obtenus sur un guide $\mathrm{PE}_{1}\left(1 \%\right.$ B.L., $\left.300^{\circ} \mathrm{C}, 5 \mathrm{~h}\right)$, recuit en plusieurs paliers de une ou deux heures à $330^{\circ} \mathrm{C}$ et $350^{\circ} \mathrm{C}$. Les photos sur la droite sont celles du faisceau réfléchi prises aux différentes positions repérées par des chiffres. La photo correspondant au spot en 3 n'est pas représentée, mais elle est identique à la photo $n^{\circ} 2$ de la figure 4.6. 
forte dégradation de la qualité du spot. L'observation au microscope de la face des échantillons indique qu'ils sont de qualité équivalente, et nous pensons donc que la différence des spots réfléchis s'explique par la qualité cristalline de la couche échangée ou par celle de l'interface entre le guide et le substrat. En dépit de ces difficultés d'interprétation, ces résultats indiquent cependant que le coefficient non linéaire est au moins restauré dans la partie initialement échangée. Nous pensons que cela est dû au fait que le guide initial est réalisé en phase $\beta_{1}$, et non pas en phase $\beta_{2}$, qui est celle que l'on obtient en utilisant les paramètres d'échange choisis par les autres auteurs $[3,4,5]$ (acide benzoïque pur à $200^{\circ} \mathrm{C}$ ). Des études plus approfondies sont en cours pour tenter d'éclaircir ces points et d'éviter la dégradation du faisceau réfléchi en utilisant d'autres traitements thermiques pour le recuit.

\subsection{Structure des domaines dans les guides réalisés par échange protonique sur niobate de lithium}

Dans ce paragraphe, nous allons étudier l'influence de l'échange protonique sur l'organisation périodique des domaines dans le PPLN et montrer que le processus de type $\mathrm{PE}_{\mathrm{II}}[65]$ qui permet de respecter le coefficient non linéaire permet également de respecter l'organisation

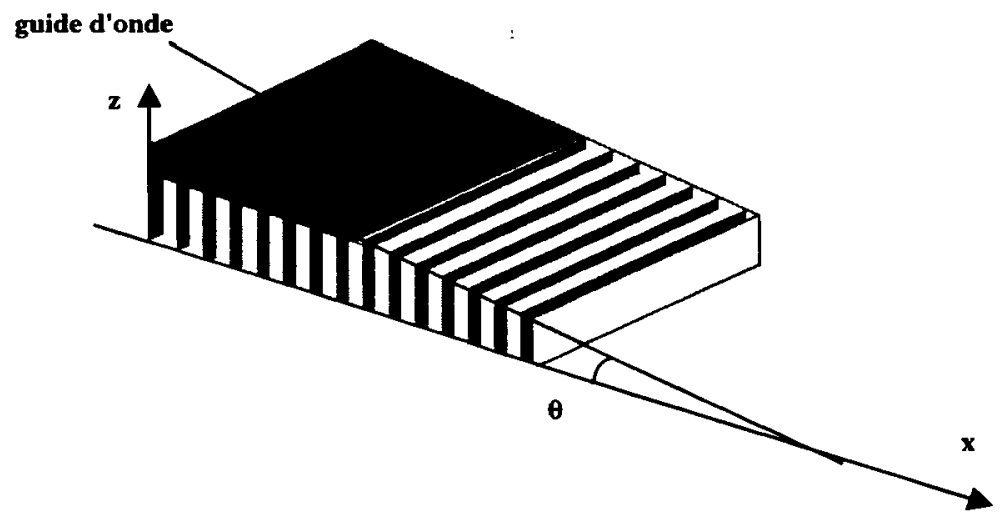

Figure 4.8 : Schéma des échantillons de PPLN échangés et polis avec un faible angle par rapport à la surface initiale

périodique. Pour cette étude, nous avons préparé des guides $\mathrm{PE}_{1}$ et $P E_{\mathrm{II}}$ identiques à ceux qui ont été utilisés pour les mesures du coefficient non linéaire. Le guide recuit avait été échangé pendant $25 \mathrm{mn}$ dans un bain à $300^{\circ} \mathrm{C}$ contenant $1 \%$ de benzoate de lithium et recuit pendant $6 \mathrm{~h}$ à $350^{\circ} \mathrm{C}$ pour atteindre la phase $\alpha$. Les surfaces de ces échantillons ont ensuite étaient polies en inclinant le plan de polissage d'environ $0,3^{\circ}$ par rapport à la surface initiale (figure 4.8). Ceci permet en observant l'échantillon par dessus, d'observer à la fois les régions ayant subi l'échange et les régions restées vierges. Une attaque chimique à l'aide d'un mélange $\mathrm{HF} / \mathrm{HNO}_{3}$ qui attaque beaucoup moins vite les zones orientées $\mathrm{Z}^{*}$ que les zones orientées $Z$ permet ensuite de révéler l'orientation des domaines [66] . Les figures 4.9 à 4.11 sont les photos après attaque des trois échantillons étudiés.

Pour le guide $\mathrm{PE}_{\mathrm{I}}$ (figure 4.9), toute la partie de l'échantillon qui correspond à la zone échangée ne présente plus de structure périodique, ce qui montre que le processus d'échange a effacé cette organisation. 


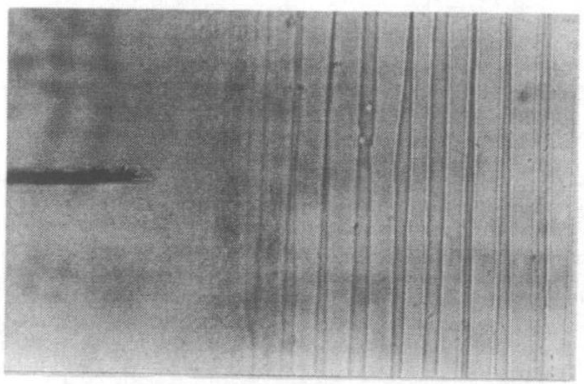

Figure 4.9. Vue de dessus, après polissage en biais et attaque chimique d'un échantillon de PPLN ayant subi un échange protonique de type $\mathrm{PE}_{1}$. La partie échangée ne présente plus de structure périodique.

Pour le guide recuit (figure 4.10), la photo est similaire à la précédente, ce qui montre que le processus de recuit standard que nous avons utilisé pour cet échantillon ne permet pas au cristal de retrouver la structure périodique présente dans le substrat. Cependant cette expérience ne permet pas de dire si les domaines ont été effacés sur toute la profondeur du guide ou simplement sur une partie de celle-ci.

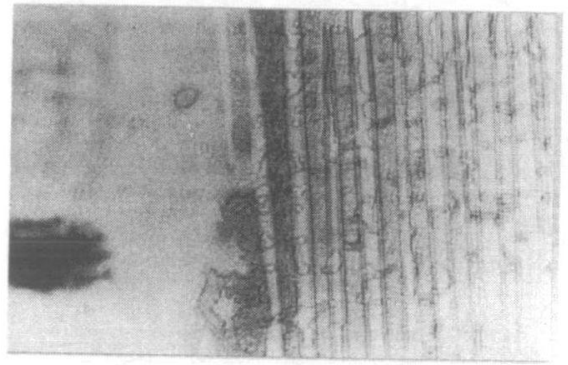

Figure 4.10. Vue de dessus, après polissage en biais et attaque chimique d'un échantillon de PPLN ayant subi un échange protonique de type PE, suivit d'un recuit. La partie échangée ne présente plus de structure périodique.

Enfin, dans le cas du guide $\mathrm{PE}_{\mathrm{m}}$ (figure 4.11), on voit que les domaines existent sur toute la surface de l'échantillon, échangé ou non. Ce processus de fabrication qui n'entraîne aucun changement de phase cristalline permet donc de réaliser des guides sans effacer la structure périodique du PPLN.

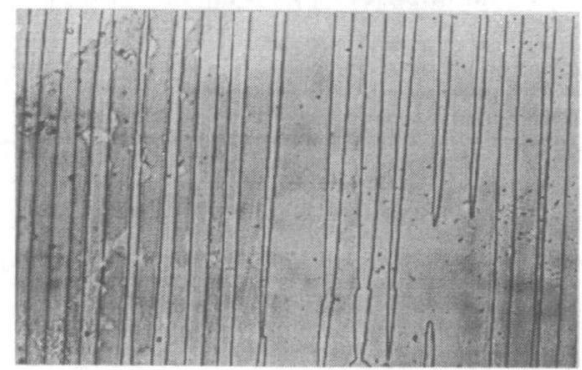

Figure 4.11. Vue de dessus, après polissage en biais et attaque chimique d'un échantillon de PPLN ayant subi un échange protonique de type $\mathrm{PE}_{111}$. La partie échangée présente une structure périodique intacte. 


\subsection{Efficacité de conversion}

Ces résultats permettent de comprendre pourquoi les générateurs et les oscillateurs paramétriques réalisés jusqu'à présent en configuration optique intégrée présentent des efficacités inférieures à celles qui sont prédites théoriquement, alors que les versions "optique massive" atteignent fréquemment les performances prédites. En effet, depuis 1993, on est passé des substrats polarisés par diffusion de titane aux substrats polarisés par champ électrique ce qui a permis d'améliorer le recouvrement et de gagner deux ordres de grandeur sur l'efficacité de conversion dans les expériences de fluorescence paramétrique [67]. Cependant, le seuil d'oscillation le plus bas obtenu pour un OPOI (Oscillateur Paramétrique Optique Intégré) est de l'ordre de 1,5 W alors qu'il ne devrait pas dépasser les $10 \mathrm{~mW}$ pour un composant optimisé. De même les générateurs de fréquence basés sur le principe de la différence de fréquence présentent des rendements de $375 \% \mathrm{~W}^{-1}$, alors que la théorie prévoit $800 \% \mathrm{~W}^{-1}$ pour un guide de cette longueur.

Tous ces composants ont en effet été réalisés en utilisant la technique d'échange plus recuit pour créer les guides d'onde, ce qui perturbe la structure des domaines. Ce degré de perturbation dépend fortement des paramètres utilisés pour fabriquer le guide, mais d'une façon qu'il est difficile de prévoir a priori. Notre étude de l'influence des différents types d'échange protonique sur le coefficient non linéaire et sur l'organisation des domaines, permet de penser qu'il y a un moyen de réaliser un OPOI présentant les performances prédites théoriquement. Il ne reste plus qu'à résoudre le problème technologique posé par la mise au point d'un processus de masquage compatible avec un échange de type $\mathrm{PE}_{\mathrm{III}}$. Il y a cependant encore beaucoup de travail à faire pour comprendre complètement l'influence de l'échange et des recuits sur la forme des domaines.

\section{CONCLUSION}

La disponibilité de cristaux non linéaires polarisés périodiquement a profondément modifié le domaine de l'optique non linéaire. Le nombre de publications relatant la réalisation de composants faisant appel à cette technique est en pleine explosion. Dans ce chapitre, nous avons mentionné un certain nombre de ces réalisations qui devraient permettre à l'optique non linéaire de sortir du domaine des laboratoires pour trouver des applications dans le domaine des télécommunications, ou des capteurs basés sur des analyses spectroscopiques.

À côté de l'amélioration des performances des composants qui ont été réalisés dans les laboratoires et de leur éventuelle industrialisation, la communauté scientifique doit encore faire face à deux défis importants :

- la réalisation de composants non linéaires en optique intégrée pour bénéficier du confinement de l'énergie et réaliser des composants non linéaires fonctionnant avec très peu de puissance

- la mise au point de nouveaux matériaux non linéaires plus performants ou couvrant d'autres parties du spectre.

\section{Références}

[1] Webjörn J., Pruneri V., Russel P, Barr J.R.M. et Hanna D.C., "Blue light generation in bulk lithium niobate electrically poled via liquid electrode", Electron. Lett.30 (1994) 894

[2] Amstrong J. A., Bloembergen N., Ducuing J. et Pershan P.S., "Interactions between light waves in nonlinear dielectric", Phys. Rev.,127 (1962) 1918-1939 
[3] Lim E.J., Fejer M.M. et Byer R.L., "Second harmonic generation of green light in periodically poled lithium niobate waveguide", Electron. Lett., 25 (1989) 174

[4] Webjörn J., Laurell F. et Arvidsson G., "Blue light generated by frequency doubling of laser diode light in a lithium niobate waveguide", IEEE Photonics Tech. Lett., 1 (1989) 316-318

[5] Myers L.E., Eckardt R.C., Fejer M.M, Byer R.L. et Pierce J.W., "CW-diode pumped optical parametric oscillator in bulk periodically poled $\mathrm{LiNbO}_{3}$ ", Electronics Letters, 31 $N^{\circ} 21$ (1995) 1869-1870

[6] Bosenberg W.R., Dobshoff A., Alexander J.I., Myers L E. et Byer R.L., "Continouswave singly resonnant optical parametric oscillator based on periodically poled $\mathrm{LiNbO}_{3}$ " Optics Letters, 21 (10) (1996) 713-715

[7] Jackel J.L., Rice R.E. et Veslka J.J., "Proton exchange for high index waveguides in LiNbO3", Appl. Phy. Lett., 41 (1982) 607

[8] De Micheli M., Botineau J., Neveu S., Sibillot P., Ostrowsky D.B. et Papuchon M. "Independant control of index and profiles in proton exchanged lithium niobate guides" Opt. Lett., 8 (1983) 114-115

[9] Bierlein J.D., Laubacher D.B., Brown J.B., van der Poel C.J., "Balanced phase matching in segmented $\mathrm{KTiOPO}_{4}$ waveguides." Appl. Phys. Lett. 56 (18) (1990) 1725-1727,

[10] Okada M., Takizawa K., Ieiri S., " Second harmonic generation by periodic laminar structure of nonlinear optical crystal", Opt. Comm. 18(3) (1976) 331-334,

[11] Eger D., Oron M.., Katz M. , Zussman A, "Highly efficient blue light generation in $\mathrm{KTiOPO}_{4}$ waveguides", Appl. Phys. Lett. 64(24) (1994) 3208-3209

[12] Webjorn J., Laurell F., Arvidsson G., " Fabrication of periodically domain inverted channel waveguides in lithium niobate for second harmonic generation.", J. Light. Tech. 7(10) (1989) 1597-1600

[13] cf paragraphe 3.1

[14] Armani F., Delacourt D., Lallier E., Papuchon M., He Q., De Micheli M., Ostrowsky D.B., "First order quasi-phase matched $\mathrm{LiNbO}_{3}$ waveguides."Electron. Lett. 28 (1992) 139-140

[15] Yamada M., Nada N.,Saitoh M., Watanabe K., "First-order quasi-phase matched $\mathrm{LiNbO}_{3}$ waveguide periodically poled by applying an external field for efficient blue secondharmonic generation.", Appl. Phys. Lett. 62 (5) (1993) 435-436

[16] Miller G.D., Batchko R.G., Fejer M.M., Byer R.L., "Visible quasi-phase matched harmonic generation by electric-field-poled lithium niobate", SPIE Proc. Vol. 2700, (1996) 34-45

[17] Edwards T.J., Turnbull G.A.; Dunn M.H., Ebrahimzadeh M., Karlson H., Ardvidson G., Laurell F., "Continuous-wave, singly resonant optical parametric oscilator based on periodically poled RbTiOAsO 4 . », CLEO’98, paper CMK1, San Francisco, May 98

[18] Batchko R.B., Weise D.R., Plettner T, Miller G.D., Fejer M.M., Byer R.L., "Continuouswave $532 \mathrm{~nm}$ pumped singly resonant optical parametric oscillator based on periodically poled lithium niobate.", Opt. Lett. 23 (3) (1998) 168-170

[19] Myers L.E., Eckardt R.C., Fejer M.M., Byer R.L., Bosenberg W.R., Pierce J.W., J. Opt. Soc. Am. B 12 (1995) 2102)

[20] Lindsay I.D., Turnbull G.A.,. Dunn M.H, Ebrahimzadeh M., "Doubly resonant cw PPLN optical parametric oscillator pumped by injection-locked broad area diode laser.", CLEO'98, paper CMK3, San Francisco, May 98 
[21] Klein M.E.,.Lee D.H, Meyn. J.P.,.Beier B, Boller K.J., Wallenstein R., “Diode pumped $\mathrm{cw}$ optical parametric oscillator with large temperature tunability based on periodically poled lithium tantalate" CLEO'98, paper CMK2, San Francisco, May 98

[22] Schneider K., Kramper P., de Natale P., Inguscio M., Schiller S., Mlyneck J., "Widely tunable parametric iscillator for high resolution spectroscopy ", CLEO'98, paper CMK5, San Francisco, May 98

[23] Fejer M.M., Magel G.A., Jundt D.H., Byer R.L., “ Quasi-phase-matched second harmonic generation : tuning and tolerances.", J. Quant. Elec. 28 (11) (1992) 2631-2654)

[24] Becouarn L., Lallier E., Brévignon M., Lehoux J., “ Cascaded second-harmonic and sum-frequency generation of a $\mathrm{CO}_{2}$ laser using a single quasi-phase-matched $\mathrm{GaAs}$ crystal." CLEO'98, paper CTUM23, San Francisco, May 98

[25] Galvanauskas A., Hariharan A., Harter D.,. Arbore M.A, Fejer M.M., “ Micro laser pumped, engineerable bandwidth parametric chirped-pulse amplifier using electric-fieldpoled $\mathrm{LiNbO}_{3}$ ", CLEO'98, paper CME2, San Francisco, May 98

[26] Reid D.T., Loza-Alvarez P., Ebrahimzadeh M., Rafailov E.U., Faller P., Birkin D.J., Sibett W., Karlson H., Laurell F., “ Femtosecond pulse compression by second harmonic generation in aperiodically poled KTP.", CLEO'98, paper CME3, San Francisco, May 98

[27] Boyd J.T., (1972) J.Q.E. 8788

[28] Bloembergen N., " Concervation laws in nonlinear optics", J. Opt. Soc. Am. 70 (12) (1980) 1429-1436

[29] Stegeman G.I. et Seaton C.T., "Nonlinear integrated optics", J. Appl. Phys 58 (12) (1985). R57-R78

[30] Hayata K., Sugawara T. et Koshiba M. "Modal analysis of second harmonic electromagnetic field generated by the Cerenkov effect in optical waveguisdes.", (1990), J. of Quan. Elect. 26 (1) 123-134

[31] Yariv A. "Quantum Electronics",(1975), Wiley, New York

[32] Ito $H$. and Inaba H., "Efficient phase matched second harmonic generation method in four layered optical waveguide structures.", Opt. Lett. 2 (6) (1978) 139-141

[33] De Micheli M., Botineau J., Sibillot P., Neveu S., Ostrowsky D.B. et Papuchon M., "Extention of second harmonic phase matching range in Lithium Niobate guides", Opt. Lett. 8 (1983) 116-118

[34] Schmidt R.V.et Kaminov I.P., " Metal diffused optical waveguides in $\mathrm{LiNbO}_{3}$." Appl. Phys. Lett. 25 (8) (1974) 458

[35] Sohler W. p. 449, et Suche H. p. 480. "New directions in Guided Waves and Coherent Optics", Ed. By D.B. Ostrowsky and E. Spitz, Martinus Nijhoff Ed., The Hague (1984),

[36] Hermann H. et Sohler W., "Difference-frequency generation of tunable, coherent midinfrared radiation in Ti:LiNbO3 channel waveguides.", J. Opt. Soc. Am. B 5 (2) (1988) 267-277

[37] He Q., De Micheli M.P, Ostrowsky D.B., Lallier E., Pocholle J.P., Papuchon M., Armani F., Delacourt D.,. C. Grezes-Besset C.et Pelletier E.; "Self-frequency-doubled high $\delta$ proton exchanged $\mathrm{Nd}: \mathrm{LiNbO}_{3}$ waveguide laser, Opt. Comm 89 (1) (1992) 54-58

[38] Li M.J., De Micheli M.P. et Ostrowsky D.B., "Cerenkov configuration Second Harmonic Generation in Proton Exchanged Lithium Niobate waveguides", J. Quant. Elect. 26 (8) (1990) 1384-1393

[39] Thyagarajan K., Mahalakshmi V. et Shenoy M.R, "Performance comparison of different configuration for second harmonic generation in planar waveguides.", Int. Journal of Optoelectronics, 8 (4) (1993) 319-332 
[40] Taniuchi T. et Yamamoto K., " Mignaturized light sources of coherent blue radiation." Proc. CLEO'87, p. 198

[41] Bierlein .D., Roelofs M.G., Brown J.B. Tohma T. et Okamoto S., " KTiOPO 4 Blue laser using segmented waveguide structures." Compact Blue-Green Lasers' 94 ,

[42] Yamada M., Nada N., Saitoh $M$ et Watanabe K., " First order quasi-phase matched $\mathrm{LiNbO}_{3}$ waveguides periodically poled applying an external field for efficient blue second harmonic generation.", Appl. Phys. Lett., 60 (1993) 435-436

[43] Fujimura M. et al. , Electron. Lett., 28 (1992) 1868-1869

[44] Delacourt D., Armani F. et Papuchon M. " Second harmonic generation efficiency in periodically poled LiNbO3 waveguides.", J. of Quan. Elect. 30 (4) (1994) 1090-1099

[45] Machio S. Nitanda F., Ito K et Sato M., " Fabrication of periodically inverted domains structures in $\mathrm{LiTaO}_{3}$ and $\mathrm{LiNbO}_{3}$ using proton exchange.", Appl. Phys. Lett. 61 (26) (1992).3077-3079

[46] Gupta M.C., Risk W.P., Nutt A.C.G. et Lau S.D., " Domain inversion in $\mathrm{KTiOPO}_{4}$ using electron beam scanning." Appl. Phys. Lett. 63 (9) (1993) 1167-1169

[47] Myers L. E., PhD dissertation (G. L. n $^{\circ}$ 5396, Stanford University) (1995),

[48] Chen Q et.. Risk W.P, " Periodic poling of $\mathrm{KTiOPO}_{4}$ using an applied electric field." Electron. Lett., 30 (18) (1994) 1516-1517

[49] Baldi P., Nouh S., De Micheli M.P., Ostrowsky D.B., Delacourt D. Banti X. et Papuchon M., Efficient quasi phase-matched generation of parametric fluorescence in room temperature lithium niobate waveguides, Elect. Lett. 29 (17) (1993) 1539

[50] Baldi P., Aschieri P., Nouh S., De Micheli M. et Ostrowsky D.B.; Delacourt D. et Papuchon M., " Modelling and experimental observation of parametric fluorescence in periodically poled lithium niobate waveguides. " J. of Quan. Elect. 31 n $^{\circ} 6$ (1995) 997 1008

[51] Mueller C.T., et Garmire E., "Photorefractive effect in $\mathrm{LiNbO}_{3}$ directional couplers", App. Opt., 23 (1984) 4348-4351

[52] Baron C., Cheng H. et Gupta M.C., " Domain inversion in $\mathrm{LiTaO}_{3}$ and $\mathrm{LiNbO}_{3}$ by electric field application on chemically patterned crystals.", Appl. Phys. Lett. 68 (4) (1996) 22

[53] Aboud I., De Micheli M. et Ostrowsky D.B.; Smith P.G.R. et Hanna D., " Etude de l'influence de la fabrication des guides d'ondes sur l'inversion de la polarisation dans le niobate de lithium ", JNOG'97 - Saint Etienne - France

[54] Korkishko Yu. N,. Fedorov V.A, De Micheli M., El Hadi K., Baldi P., Leycuras A., "Relationships between structural and optical properties of proton-exchnaged waveguides on Z-cut lithium niobate.", Applied Optics, $35 \mathrm{~N}^{\circ} 36$ (1996) 7056-7060

[55] White J.M. et Heidrich P.F., "Optical waveguide refractive index profiles determined from the measurement of mode indices: a simple analysis", Appl. Opt. 15 (1976) 151

[56] Fedorov V.A., Ganshin V.A. et Korkishko Yu. N., " New method of double-crystal XRay diffractometric determination of the strained state in surface-layer structures.", Phys. Status Solidi (a) 135(1993) 493

[57] El Hadi K., Baldi P., Nouh S.. De Micheli M P, Leycuras A., Fedorov V A. et Korkishko Yu. N., "Control of proton echange for $\mathrm{LiTaO}_{3}$ waveguides and crystal structure of $\mathrm{H}_{\mathrm{x}} \mathrm{Li}_{1-\mathrm{x}} \mathrm{TaO}_{3}$ ", Opt. Lett. 20 (16) (1995) 1698-1700

[58] Chen S., De Micheli M.P., Baldi P., Ostrowsky D.B., Leycuras A., Tartarini G. et Bassi P., "Hybrid modes in Proton Exchanged waveguides realized in $\mathrm{LiNbO}_{3}$, and their dependance on Fabrication parameters", J. Light. Tech. 12 (5) (1994) 862-871

[59] Chen S., PhD dissertation, Nice 1992 
[60] Bortz M.L., Eyres L.A. et Fejer M.M., "Depth profiling of the d33 nonlinear coefficient in annealed proton exchanged $\mathrm{LiNbO}_{3}$ waveguides", Appl. Phys. Lett. 62 2012-2014

[61] Cao X., Srivastava R., Ramaswamy R.V. et Natour J., "Recovery of second order optical nonlinearity in annealed proton-exchanged LiNbO3", Photon. Technol. Lett. 3, (1993)

[62] Laurell F., Roelofs M.G, et Hsiung H., "Loss of optical nonlinearity in proton-exchanged LiNbO3 waveguides", Appl. Phys. Lett. 60 (1992) 301-303

[63] El Hadi K., Sundheimer M., Aschieri P., Baldi P., De Micheli M.P. et Ostrowsky D.B.; Laurell F., "Quasi-phase-matched parametric interactions in proton exchanged lithium niobate waveguides", J. of Opt. Soc. of Ame. B, 14 (11) (1997) 3197-3203

[64] Ahlfeldt H., "Non-linear optical properties of proton-exchanged waveguides in Z-cut LiTaO3", J Appl. Phys. 76 (6) (1994) 3255-3260

[65] Li M.J., De Micheli M., Ostrowsky D., et Papuchon M., " Fabrication et caractérisation des guides PE présentant une faible variation d'indice et une excellente qualité optique", J. Optics (Paris) 18 (3) (1987) 139-144

[66] Nassau K.,. Levinstein H.J, et Loïcano G.M., "Ferroelectric lithium niobate 1: Growth, domain structure, dislocations and etching ", J. Phys. Chem. Solids 27(1966) 983-988..

[67] Baldi P., Aschieri P., Nouh S., De Micheli M., Ostrowsky D. B., Delacourt D., et Papuchon M., "Modeling and Experimental observation of Parametric Fluorescence in Periodically Poled Lithium Niobate Waveguides", IEEE J. of Quan. Elect., 31 (6) (1995) 997-1008 